\title{
ENCYCLOPEDIA OF TYPES OF ALGEBRAS 2010
}

\author{
G. W. ZINBIEL
}

\begin{abstract}
This is a cornucopia of types of algebras with some of their properties from the operadic point of view.
\end{abstract}

\section{INTRODUCTION}

The following is a list of some types of algebras together with their properties under an operadic and homological point of view. We keep the information to one page per type and we provide one reference as Ariadne's thread. More references are listed by the end of the paper. We work over a fixed field $\mathbb{K}$ though in many instances everything makes sense and holds over a commutative ground ring. The category of vector spaces over $\mathbb{K}$ is denoted by Vect. All tensor products are over $\mathbb{K}$ unless otherwise stated.

The items of a standard page (which is to be found at the end of this introduction) are as follows.

Sometimes a given type appears under different names in the literature. The choice made in Name is, most of the time, the most common one (up to a few exceptions). The other possibilities appear under the item Alternative.

The presentation given in Definit. is the most common one (with one exception). When others are used in the literature they are given in Alternative. The item oper. gives the generating operations. The item sym. gives their symmetry properties, if any. The item rel. gives the relation(s). They are supposed to hold for any value of the variables $x, y, z, \ldots$. If, in the presentation, only binary operations appear, then the type is said to be binary. Analogously, there are ternary, $k$-ary, multi-ary types.

If, in the presentation, the relations involve only the composition of two operations at a time (hence 3 variables in the binary case, 5 variables in the ternary case), then the type is said to be quadratic.

For a given type of algebras $\mathcal{P}$ the category of $\mathcal{P}$-algebras is denoted by $\mathcal{P}$-alg. For each type there is defined a notion of free algebra. By definition the free algebra of type $\mathcal{P}$ over the vector space $V$ is an algebra denoted by $\mathcal{P}(V)$ satisfying the following universal condition:

for any algebra $A$ of type $\mathcal{P}$ and any linear map $\phi: V \rightarrow A$ there is a unique $\mathcal{P}$-algebra morphism $\tilde{\phi}: \mathcal{P}(V) \rightarrow A$ which lifts $\phi$. In other words the 
forgetful functor

$$
\mathcal{P} \text {-alg } \longrightarrow \text { Vect }
$$

admits a left adjoint

$$
\mathcal{P}: \text { Vect } \longrightarrow \mathcal{P} \text {-alg. }
$$

In all the cases mentioned here the relations involved in the presentation of the given type are (or can be made) multilinear. Hence the functor $\mathcal{P}(V)$ is of the form (at least in characteristic zero),

$$
\mathcal{P}(V)=\bigoplus_{n \geq 1} \mathcal{P}(n) \otimes_{\mathbb{S}_{n}} V^{\otimes n},
$$

where $\mathcal{P}(n)$ is some $\mathbb{S}_{n}$-module. The $\mathbb{S}_{n}$-module $\mathcal{P}(n)$ is called the space of $n$-ary operations since for any algebra $A$ there is a map

$$
\mathcal{P}(n) \otimes_{\mathbb{S}_{n}} A^{\otimes n} \rightarrow A .
$$

The functor $\mathcal{P}:$ Vect $\rightarrow$ Vect inherits a monoid structure from the properties of the free algebra. Hence there exist transformations of functors $\iota:$ Id $\rightarrow \mathcal{P}$ and $\gamma: \mathcal{P} \circ \mathcal{P} \rightarrow \mathcal{P}$ such that $\gamma$ is associative and unital. The monoid $(\mathcal{P}, \gamma, \iota)$ is called a symmetric operad.

The symmetric operad $\mathcal{P}$ can also be described as a family of $\mathbb{S}_{n}$-modules $\mathcal{P}(n)$ together with maps

$$
\gamma\left(i_{1}, \ldots, i_{k}\right): \mathcal{P}(k) \otimes \mathcal{P}\left(i_{1}\right) \otimes \cdots \otimes \mathcal{P}\left(i_{k}\right) \longrightarrow \mathcal{P}\left(i_{1}+\cdots+i_{k}\right)
$$

satisfying some compatibility with the action of the symmetric group and satisfying the associativity property.

If $\mathbb{S}_{n}$ is acting freely on $\mathcal{P}(n)$, then $\mathcal{P}(n)=\mathcal{P}_{n} \otimes \mathbb{K}\left[\mathbb{S}_{n}\right]$ where $\mathcal{P}_{n}$ is some vector space, and $\mathbb{K}\left[\mathbb{S}_{n}\right]$ is the regular representation. If, moreover, the maps $\gamma\left(i_{1}, \ldots, i_{n}\right)$ are induced by maps

$$
\gamma_{i_{1}, \ldots, i_{k}}: \mathcal{P}_{k} \otimes \mathcal{P}_{i_{1}} \otimes \cdots \otimes \mathcal{P}_{i_{k}} \longrightarrow \mathcal{P}_{i_{1}+\cdots+i_{k}}
$$

then the operad $\mathcal{P}$ comes a from a nonsymmetric operad (abbreviated ns operad), still denoted $\mathcal{P}$.

For more terminology and details about algebraic operads we refer to [LV11].

The generating series of the operad $\mathcal{P}$ is defined as

$$
f^{\mathcal{P}}(t):=\sum_{n \geq 1} \frac{\operatorname{dim} \mathcal{P}(n)}{n !} t^{n},
$$

in the binary case. When dealing with a nonsymmetric operad it becomes

$$
f^{\mathcal{P}}(t):=\sum_{n \geq 1} \operatorname{dim} \mathcal{P}_{n} t^{n} .
$$

The Koszul duality theory of associative algebras has been extended to binary quadratic operads by Ginzburg and Kapranov, cf. [GK94], then to quadratic operads by Fresse, cf. [Fre06]. We give a conceptual treatment of this theory, together with applications in [LV11]. So, to any quadratic operad $\mathcal{P}$, there is associated a quadratic dual operad denoted $\mathcal{P}^{!}$. It is 
often a challenge to find a presentation of $\mathcal{P}^{!}$out of a presentation of $\mathcal{P}$. One of the main results of the Koszul duality theory of operads is to show the existence of a natural differential map on the composite $\mathcal{P}^{! *} \circ \mathcal{P}$ given rise to the Koszul complex. If it is acyclic, then $\mathcal{P}$ is said to be Koszul. One can show that, if $\mathcal{P}$ is Koszul, then so is $\mathcal{P}^{!}$. In this case the generating series are inverse to each other for composition, up to sign, that is:

$$
f^{\mathcal{P}^{!}}\left(-f^{\mathcal{P}}(-t)\right)=t
$$

In the $k$-ary case we introduce the skew-generating series

$$
g^{\mathcal{P}}(t):=\sum_{n \geq 1}(-1)^{k} \frac{\operatorname{dim} \mathcal{P}((k-1) n+1)}{n !} t^{((k-1) n+1)} .
$$

If the operad $\mathcal{P}$ is Koszul, then (cf. [Val07])

$$
f^{\mathcal{P}^{!}}\left(-g^{\mathcal{P}}(-t)\right)=t .
$$

The items Free alg., rep. $\mathcal{P}(n)$ or $\mathcal{P}_{n}, \operatorname{dim} \mathcal{P}(n)$ or $\operatorname{dim} \mathcal{P}_{n}$, and Gen. series speak for themselves.

Koszulity of an operad implies the existence of a small chain complex to compute the (co)homology of a $\mathcal{P}$-algebra. When possible, the information on it is given in the item Chain-cplx. Moreover it permits us to construct the notion of $\mathcal{P}$-algebra up to homotopy, whose associated operad, which is a differential graded operad, is denoted $\mathcal{P}_{\infty}$.

The item Properties lists the main features of the operad. Set-theoretic means that there is a set operad $\mathcal{P}_{\text {Set }}$ (monoid in the category of $\mathbb{S}$-Sets) such that $\mathcal{P}=\mathbb{K}\left[\mathcal{P}_{\text {Set }}\right]$. Usually this property can be read on the presentation of the operad: no algebraic sums.

In the item Relationship we list some of the ways to obtain this operad under some natural constructions like tensor product (Hadamard product) or Manin products (white $O$ or black $\boldsymbol{0}$ ), denoted $\square$ and $\boldsymbol{\square}$ in the nonsymmetric framework, cf.[LV11]) for instance. We also list some of the most common functors to other types of algebras. Keep in mind that a functor $\mathcal{P} \rightarrow \mathcal{Q}$ induces a functor $\mathcal{Q}$-alg $\rightarrow \mathcal{P}$-alg on the categories of algebras.

Though we describe only algebras without unit, for some types there is a possibility of introducing an element 1 which is either a unit or a partial unit for some of the operations, see the discuission in [Lod04]. We indicate it in the item Unit.

For binary operads the opposite type consists in defining new operations by $x \cdot y=y x$, etc. If the new type is isomorphic to the former one, then the operad is said to be self-opposite. When it is not the case, we mention whether the given type is called right or left in the item Comment.

In some cases the structure can be "integrated". For instance Lie algebras are integrated into Lie groups (Lie third problem). If so, we indicate it in the item Comment.

In the item Ref . we indicate a reference where information on the operad and/or on the (co)homology theory can be obtained. It is not necessarily the 
first paper in which this type of algebras first appeared. For the three graces As, Com, Lie, the classical books Cartan-Eilenberg "Homological Algebra" and MacLane "Homology" are standard references.

Here is the list of the types included so far (with page number and letter $\mathrm{K}$ indicating that they are Koszul dual to each other):

\begin{tabular}{|c|c|c|c|c|}
\hline sample & 6 & As & 7 & self-dual \\
\hline Com & 8 & Lie & 9 & $\mathrm{~K}$ \\
\hline Pois & 10 & none & 11 & self-dual \\
\hline$L e i b$ & 12 & Zinb & 13 & $\mathrm{~K}$ \\
\hline Dend & 14 & Dias & 15 & K \\
\hline PreLie & 16 & Perm & 17 & K \\
\hline Dipt & 18 & Dipt! & 19 & K \\
\hline $2 a s$ & 20 & $2 a s^{!}$ & 21 & K \\
\hline Tridend & 22 & Trias & 23 & K \\
\hline PostLie & 24 & ComTrias & 25 & K \\
\hline$C T D$ & 26 & $C T D^{!}$ & 27 & K \\
\hline Gerst & 28 & $B V$ & 29 & \\
\hline$M a g$ & 30 & $\mathrm{Nil}_{2}$ & 31 & $\mathrm{~K}$ \\
\hline ComMag & 32 & ComMag! & 33 & $\mathrm{~K}$ \\
\hline Quadri & 34 & Quadri! & 35 & K \\
\hline Dup & 36 & Dup! & 37 & K \\
\hline$A s^{(2)}$ & 38 & $A s^{\langle 2\rangle}$ & 39 & \\
\hline$L$-dend & 40 & Lie-adm & 41 & \\
\hline PreLiePerm & 42 & Altern & 43 & \\
\hline Param1rel & 44 & MagFine & 45 & \\
\hline GenMag & 46 & $N A P$ & 47 & \\
\hline Moufang & 48 & Malcev & 49 & \\
\hline Novikov & 50 & DoubleLie & 51 & \\
\hline DiPreLie & 52 & Akivis & 53 & \\
\hline Sabinin & 54 & Jordan triples & 55 & \\
\hline$t-A s^{(3)}$ & 56 & $p-A s^{(3)}$ & 57 & \\
\hline$L T S$ & 58 & Lie-Yamaguti & 59 & \\
\hline Interchange & 60 & HyperCom & 61 & \\
\hline$A_{\infty}$ & 62 & $C_{\infty}$ & 63 & \\
\hline$L_{\infty}$ & 64 & $\operatorname{Dend}_{\infty}$ & 65 & \\
\hline $\mathcal{P}_{\infty}$ & 66 & Brace & 67 & \\
\hline$M B$ & 68 & 2 Pois & 69 & \\
\hline$\Xi^{ \pm}$ & 70 & your own & 71 & \\
\hline
\end{tabular}




\section{Notation}

We use the notation $\mathbb{S}_{n}$ for the symmetric group. Trees are very much in use in the description of operads. We use the following notation:

$-P B T_{n}$ is the set of planar binary rooted trees with $n-1$ internal vertices (and hence $n$ leaves). The number of elements of $P B T_{n}$ is the Catalan number $c_{n}=\frac{1}{n+1}\left(\begin{array}{c}2 n \\ n\end{array}\right)$.

$-P T_{n}$ is the set of planar rooted trees with $n$ leaves, whose vertices have valency greater than $1+2$ (one root, at least 2 inputs). So we have $P B T_{n} \subset$ $P T_{n}$. The number of elements of $P T_{n}$ is the super Catalan number, also called Schröder number, denoted $C_{n}$.

A planar binary rooted tree $t$ is completely determined by its right part $t^{r}$ and its left part $t^{l}$. More precisely $t$ is the grafting of $t^{l}$ and $t^{r}$ :

$$
t=t^{l} \vee t^{r} \text {. }
$$

\section{Comments}

Many thanks to Walter Moreira for setting up a software which computes the first dimensions of the operad from its presentation.

We remind the reader that we can replace the symmetric monoidal category Vect by many other symmetric monoidal categories. So there are notions of graded algebras, differential graded algebras, twisted algebras, and so forth. In the graded cases the Koszul sign rule is in order. Observe that there are also operads where the operations may have different degree (operad encoding Gerstenhaber algebras for instance).

We end this paper with a tableau of integer sequences appearing in this document.

This list of types of algebras is not as encyclopedic as the title suggests. We put only the types which are defined by a finite number of generating operations and whose relations are multilinear. Moreover we only put those which have been used some way or another. You will not find the "restricted types" (like divided power algebras), nor bialgebras.

Please report any error or comment to: $\quad$ gw.zinbiel@free.fr

We plan to update this encyclopedia every now and then. 
Name

Notation

Def. oper. sym. rel.

Free alg.

rep. $\mathcal{P}(n)$

$\operatorname{dim} \mathcal{P}(n)$

Gen. series

Dual operad

Chain-cplx

Properties

Alternative

Relationship

some of the relationships with other operads, either under some construction like symmetrizing, Hadamard product, Manin products, or under the existence of functors

Unit

Comment

Ref .

\section{Type of AlgeBras}

\section{Most common terminology}

my favorite notation for the operad (generic notation: $\mathcal{P}$ )

list of the generating operations

their symmetry if any

the relation(s)

the free algebra as a functor in $V$

the $\mathbb{S}_{n}$-representation $\mathcal{P}(n)$ and/or the space $\mathcal{P}_{n}$ if nonsymmetric

the series (if close formula available), the list of the 7 first numbers beginning at $n=1$

close formula for $f^{\mathcal{P}}(t)=\sum_{n \geq 1} \frac{\operatorname{dim} \mathcal{P}(n)}{n !} t^{n}$ when available

the Koszul dual operad

Explicitation of the chain complex, if not too complicated

among: nonsymmetric, binary, quadratic, set-theoretic, ternary, multi-ary, cubic, Koszul.

alternative terminology, and/or notation, and/or presentation.

whether one can assume the existence of a unit (or partial unit)

whatever needs to be said which does not fit into the other items

a reference, usually dealing with the homology of the $\mathcal{P}$-algebras

(not necessarily containing all the results of this page) 
Name

Notation

Def. oper.

sym.

rel. $\quad(x y) z=x(y z)$, operadically $\mu \circ_{1} \mu=\mu \circ_{2} \mu$ (associativity)

Free alg. $\quad A s(V)=\bar{T}(V)=\oplus_{n \geq 1} V^{\otimes n} \quad$ tensor algebra (noncommutative polynomials)

$\left(x_{1} \ldots x_{p}\right)\left(x_{p+1} \ldots x_{p+q}\right)=x_{1} \ldots x_{p+q}$ (concatenation)

rep. $\mathcal{P}(n) \quad A s(n)=\mathbb{K}\left[\mathbb{S}_{n}\right]$ (regular representation), $\quad A s_{n}=\mathbb{K}$

$\operatorname{dim} \mathcal{P}(n) \quad 1,2,6,24,120,720,5040, \ldots, n !, \ldots$

Gen. series $f^{A s}(t)=\frac{t}{1-t}$

Dual operad $A s^{!}=A s$

Chain-cplx

non-unital Hochschild complex, $C_{n}^{A s}(A)=A^{\otimes n}$

$b^{\prime}\left(a_{1}, \ldots, a_{n}\right)=\sum_{i=1}^{i=n-1}(-1)^{i-1}\left(a_{1}, \ldots, a_{i} a_{i+1}, \ldots, a_{n}\right)$

important variation: cyclic homology

Properties binary, quadratic, ns, set-theoretic, Koszul, self-dual.

Alternative Associative algebra often simply called algebra.

Can be presented with commutative operation $x \cdot y:=x y+y x$ and anti-symmetric operation $[x, y]=x y-y x$ satisfying $\left\{\begin{array}{l}{[x \cdot y, z]=x \cdot[y, z]+[x, z] \cdot y,} \\ (x \cdot y) \cdot z-x \cdot(y \cdot z)=[x,[y, z]] .\end{array}\right.$

(Livernet and Loday, unpublished)

Relationship Ass-alg $\rightarrow$ Lie-alg, $[x, y]=x y-y x$, $C o m$-alg $\rightarrow$ Ass-alg (inclusion), and many others

Unit

$1 x=x=x 1$

Comment one the three graces 
Name

Notation

Def . oper. sym. rel.

Free alg.

rep. $\mathcal{P}(n)$

$\operatorname{dim} \mathcal{P}(n)$

Gen. series

Dual operad

Chain-cplx

Properties

Alternative

Relationship

Unit

Comment

\section{Commutative algebra}

\section{Com}

$x y$

$x y=y x$ (commutativity)

$(x y) z=x(y z)$ (associativity)

$\operatorname{Com}(V)=\bar{S}(V)$ (polynomials)

if $V=\mathbb{K} x_{1} \oplus \cdots \oplus \mathbb{K} x_{n}$, then $\mathbb{K} 1 \oplus \operatorname{Com}(V)=\mathbb{K}\left[x_{1}, \ldots, x_{n}\right]$ $\operatorname{Com}(n)=\mathbb{K}$ (trivial representation)

$1,1,1,1,1,1,1, \ldots, 1, \ldots$

$f^{C o m}(t)=\exp (t)-1$

Com! $^{!}=$Lie

Harrison complex in char. 0, André-Quillen cplx in general

binary, quadratic, set-theoretic, Koszul.

sometimes called associative and commutative algebra other notation $\mathrm{Comm}$

$C o m$-alg $\rightarrow A s$-alg,

Zinb-alg $\rightarrow C o m$-alg

$1 x=x=x 1$

one of the three graces 
Name

Notation

Def. oper. $\quad[x, y]$ (bracket)

sym. $\quad[x, y]=-[y, x]$ (anti-symmetry)

rel. $\quad[[x, y], z]=[x,[y, z]]+[[x, z], y]$ (Leibniz relation)

Free alg. $\quad \operatorname{Lie}(V)=$ subspace of the tensor algebra $T(V)$ generated by $V$ under the bracket

rep. $\mathcal{P}(n) \quad$ Lie $(n)=\operatorname{Ind}_{C_{n}}^{\mathbb{S}_{n}}(\sqrt[n]{1})$

$\operatorname{dim} \mathcal{P}(n) \quad 1,1,2,6,24,120,720, \ldots,(n-1) !, \ldots$

Gen. series $f^{\text {Lie }}(t)=-\log (1-t)$

Dual operad $L i e^{!}=$Com

Chain-cplx Chevalley-Eilenberg complex $C_{n}^{\text {Lie }}(\mathfrak{g})=\Lambda^{n} \mathfrak{g}$ $d\left(x_{1} \wedge \ldots \wedge x_{n}\right)=$ $\sum_{1 \leq i<j \leq n}(-1)^{j}\left(x_{1} \wedge \ldots \wedge\left[x_{i}, x_{j}\right] \wedge \ldots \wedge \widehat{x_{j}} \wedge \ldots \wedge x_{n}\right)$

Properties binary, quadratic, Koszul.

Alternative The relation is more commonly written as the Jacobi identity: $[x,[y, z]]+[y,[z, x]]+[z,[x, y]]=0$

Relationship $A s$-alg $\rightarrow$ Lie-alg, Lie-alg $\rightarrow$ Leib-alg, PreLie-alg $\rightarrow$ Lie-alg

Unit no

Comment one of the three graces. Named after Sophus Lie. Integration: Lie groups. 
Name

Notation

Def. oper. $\quad x y,\{x, y\}$ sym. $\quad x y=y x,\{x, y\}=-\{y, x\}$

$\begin{aligned} & \text { rel. }\{\{\{x, y\}, z\}=\{x,\{y, z\}\}+\{\{x, z\}, y\} \\ &\{x y, z\}=x\{y, z\}+\{x, z\} y, \\ &(x y) z-x(y z)=0\end{aligned}$

Free alg. $\quad \operatorname{Pois}(V) \cong \bar{T}(V)$ (tensor module, iso as Schur functors)

rep. $\mathcal{P}(n) \quad$ Pois $(n) \cong \mathbb{K}\left[\mathbb{S}_{n}\right]$ (regular representation)

$\operatorname{dim} \mathcal{P}(n) \quad 1,2 !, 3 !, 4 !, 5 !, 6 !, 7 !, \ldots, n !, \ldots$

Gen. series $\quad f^{\text {Pois }}(t)=\frac{t}{1-t}$

Dual operad Pois ${ }^{!}=$Pois

Chain-cplx Isomorphic to the total complex of a certain bicomplex constructed from the action of the Eulerian idempotents

Properties $\quad$ binary, quadratic, quasi-regular, Koszul, self-dual.

Alternative Can be presented with one operation $x * y$ satisfying the relation $(x * y) * z=x *(y * z)+$

$$
\frac{1}{3}(+x *(z * y)-z *(x * y)-y *(x * z)+y *(z * x))
$$

Relationship Pois-alg $\rightleftarrows$ Lie-alg, Pois-alg $\rightleftarrows$ Com-alg,

Unit $\quad 1 x=x=x 1,[1, x]=0=[x, 1]$

Comment Named after Siméon Poisson.

Ref. [Fre06] B. Fresse, Théorie des opérades de Koszul et homologie des algèbres de Poisson, Ann.Math. Blaise Pascal 13 (2006), 237-312. 
This page is inserted so that, in the following part, an operad and its dual appear on page $2 n$ and $2 n+1$ respectively. Since Pois is self-dual there is no point to write a page about its dual.

Let us take the opportunity to mention that if $A$ is a $\mathcal{P}$-algebra and $B$ is a $\mathcal{P}$ !-algebra, then the tensor product $A \otimes B$ inherits naturally a structure of Lie algebra. If $\mathcal{P}$ is nonsymmetric, then so is $\mathcal{P}^{!}$, and $A \otimes B$ is in fact an associative algebra.

In some cases (like the Leibniz case for instance), $A \otimes B$ is a pre-Lie algebra. 
Name

Notation

Def. oper. $\quad[x, y]$

sym.

rel. $\quad[[x, y], z]=[x,[y, z]]+[[x, z], y]$ (Leibniz relation)

Free alg. $\quad \operatorname{Leib}(V) \cong \bar{T}(V)$ (reduced tensor module, iso as Schur functors)

rep. $\mathcal{P}(n) \quad$ Leib $(n)=\mathbb{K}\left[\mathbb{S}_{n}\right]$ (regular representation)

$\operatorname{dim} \mathcal{P}(n) \quad 1,2 !, 3 !, 4 !, 5 !, 6 !, 7 !, \ldots, n !, \ldots$

Gen. series $\quad f^{L e i b}(t)=\frac{t}{1-t}$

Dual operad $L e i b^{!}=$Zinb

Chain-cplx $\quad C_{n}^{L e i b}(\mathfrak{g})=\mathfrak{g}^{\otimes n}$

$d\left(x_{1}, \ldots, x_{n}\right)=\sum_{1 \leq i<j \leq n}(-1)^{j}\left(x_{1}, \ldots,\left[x_{i}, x_{j}\right], \ldots, \widehat{x_{j}}, \ldots, x_{n}\right)$

Properties binary, quadratic, quasi-regular, Koszul.

Alternative Sometimes improperly called Loday algebra.

Relationship Leib = Perm $\bigcirc$ Lie (Manin white product), see [Val08] Lie-alg $\rightarrow$ Leib-alg, Dias-alg $\rightarrow$ Leib-alg, Dend-alg $\rightarrow$ Leib-alg

Unit

no

Comment

Named after G.W. Leibniz. This is the left Leibniz algebra. The opposite type is called right Leibniz algebra. Integration : "coquecigrues" ! see for instance [Cov10]

Ref. [Lod93] J.-L. Loday, Une version non commutative des algèbres de Lie: les algèbres de Leibniz.

Enseign. Math. (2) 39 (1993), no. 3-4, 269-293. 
Name

Notation

Def. oper. $\quad x \cdot y$

sym.

rel. $(x \cdot y) \cdot z=x \cdot(y \cdot z)+x \cdot(z \cdot y) \quad$ (Zinbiel relation)

Free alg. $\quad \operatorname{Zinb}(V)=\bar{T}(V), \quad \cdot=$ halfshuffle

$x_{1} \ldots x_{p} \cdot x_{p+1} \ldots x_{p+q}=x_{1} \operatorname{sh}_{p-1, q}\left(x_{2} \ldots x_{p}, x_{p+1} \ldots x_{p+q}\right)$

rep. $\mathcal{P}(n) \quad Z i n b(n) \cong \mathbb{K}\left[\mathbb{S}_{n}\right]$ (regular representation)

$\operatorname{dim} \mathcal{P}(n) \quad 1,2 !, 3 !, 4 !, 5 !, 6 !, 7 !, \ldots, n$ !, . .

Gen. series $\quad f^{Z i n b}(t)=\frac{t}{1-t}$

Dual operad $\quad Z i n b^{!}=$Leib

Chain-cplx

Properties binary, quadratic, quasi-regular, Koszul.

Alternative $\quad Z i n b=$ ComDend (commutative dendriform algebra) Previously called dual Leibniz algebra.

Relationship $Z$ inb $=$ PreLie $\bullet$ Com, see [Val08], Zinb $=$ ComDend Zinb-alg $\rightarrow$ Com-alg, $\quad x y=x \cdot y+y \cdot x$, Zinb-alg $\rightarrow$ Dend-alg, $\quad x \prec y=x \cdot y=y \succ x$

Unit

$1 \cdot x=0, \quad x \cdot 1=x$

Comment

symmetrization of the dot product gives, not only a commutative alg., but in fact a divided power algebra. Named after G.W. Zinbiel. This is right Zinbiel algebra.

Ref . [Lod95] J.-L. Loday, Cup-product for Leibniz cohomology and dual Leibniz algebras. Math. Scand. 77 (1995), no. 2, 189-196. 
Name

Notation

Def . oper. $\quad x \prec y, x \succ y$ (left and right operation)

sym.

rel. $\left\{\begin{aligned}(x \prec y) \prec z & =x \prec(y \prec z)+x \prec(y \succ z), \\ (x \succ y) \prec z & =x \succ(y \prec z), \\ (x \prec y) \succ z+(x \succ y) \succ z & =x \succ(y \succ z) .\end{aligned}\right.$

Free alg. $\quad \operatorname{Dend}(V)=\bigoplus_{n \geq 1} \mathbb{K}\left[P B T_{n+1}\right] \otimes V^{\otimes n}$, for pb trees $s$ and $t$ : $s \prec t:=s^{l} \vee\left(s^{r} * t\right)$, and $s \succ t:=\left(s * t^{l}\right) \vee t^{r}$ where $x * y:=x \prec y+x \succ y$.

rep. $\mathcal{P}(n) \quad \operatorname{Dend}(n)=\mathbb{K}\left[P B T_{n+1}\right] \otimes \mathbb{K}\left[\mathbb{S}_{n}\right]$, so $\operatorname{Dend}_{n}=\mathbb{K}\left[P B T_{n+1}\right]$

$\operatorname{dim} \mathcal{P}(n) \quad 1,2 \times 2 !, 5 \times 3 !, 14 \times 4 !, 42 \times 5 !, 132 \times 6 !, 429 \times 7 !, \ldots, c_{n} \times n !, \ldots$ where $c_{n}=\frac{1}{n+1}\left(\begin{array}{c}2 n \\ n\end{array}\right)$ is the Catalan number

Gen. series

$f^{\operatorname{Dend}}(t)=\frac{1-2 t-\sqrt{1-4 t}}{2 t}=y, \quad y^{2}-(1-2 t) y+t=0$

Dual operad Dend ${ }^{!}=$Dias

Chain-cplx Isomorphic to the total complex of a certain explicit bicomplex

Properties binary, quadratic, ns, Koszul.

Alternative Handy to introduce $x * y:=x \prec y+x \succ y$ which is associative.

Relationship Dend $=$ PreLie $\bullet$ As, see [Val08]

Dend-alg $\rightarrow$ As-alg, $\quad x * y:=x \prec y+y \succ x$, Zinb-alg $\rightarrow$ Dend-alg, $\quad x \prec y:=x \cdot y=: y \succ x$ Dend-alg $\rightarrow$ PreLie-alg, $\quad x \circ y:=x \prec y-y \succ x$ Dend-alg $\rightarrow$ Brace-alg, $\quad$ see Ronco [Ron00]

Unit $1 \prec x=0, x \prec 1=x, \quad 1 \succ x=x, x \succ 1=0$.

Comment

dendro $=$ tree in greek. There exist many variations.

Ref.

[Lod01] J.-L. Loday, Dialgebras, Springer Lecture Notes in Math. 1763 (2001), 7-66. 
Name

Notation

Def . oper. $\quad x \dashv y, x \vdash y$ (left and right operation)

sym.

rel. $\quad\left\{\begin{array}{l}(x \dashv y) \dashv z=x \dashv(y \dashv z), \\ (x \dashv y) \dashv z=x \dashv(y \vdash z), \\ (x \vdash y) \dashv z=x \vdash(y \dashv z), \\ (x \dashv y) \vdash z=x \vdash(y \vdash z), \\ (x \vdash y) \vdash z=x \vdash(y \vdash z) .\end{array}\right.$

Free alg

$\operatorname{Dias}(V)=\bigoplus_{n \geq 1}(\underbrace{V^{\otimes n} \oplus \cdots \oplus V^{\otimes n}}_{n \text { copies }})$

noncommutative polynomials with one variable marked

rep. $\mathcal{P}(n) \quad \operatorname{Dias}(n)=\mathbb{K}^{n} \otimes \mathbb{K}\left[\mathbb{S}_{n}\right], \quad$ Dias $_{n}=\mathbb{K}^{n}$

$\operatorname{dim} \mathcal{P}(n) \quad 1,2 \times 2 !, 3 \times 3 !, 4 \times 4 !, 5 \times 5 !, 6 \times 6 !, 7 \times 7 !, \ldots, n \times n !, \ldots$,

Gen. series $f^{\text {Dias }}(t)=\frac{t}{(1-t)^{2}}$

Dual operad Dias $!=$ Dend

Chain-cplx see ref.

Properties $\quad$ binary, quadratic, ns, set-theoretic, Koszul.

Alternative Also called associative dialgebras, or for short, dialgebras.

Relationship Dias $=$ Perm $\bigcirc A s=$ Perm $\otimes A s$

As-alg $\rightarrow$ Dias-alg, $\quad x \dashv y:=x y=: x \vdash y$

Dias-alg $\rightarrow$ Leib-alg, $\quad[x, y]:=x \dashv y-x \vdash y$

Unit

Bar-unit: $x \dashv 1=x=1 \vdash x, 1 \dashv x=0=x \vdash 1$

Comment

Ref . [Lod01] J.-L. Loday, Dialgebras,

Springer Lecture Notes in Math. 1763 (2001), 7-66. 
Name

Notation

Def. oper. $\quad x \circ y$

sym.

rel.

Free alg. $\quad \operatorname{PreLie}(V)=\{$ rooted trees labeled by elements of $V\}$

rep. $\mathcal{P}(n) \quad$ PreLie $(n)=\mathbb{K}[$ rooted trees, vertices labeled by $1, \ldots, n\}]$

$\operatorname{dim} \mathcal{P}(n) \quad 1,2,9,64,625,1296,117649, \ldots, n^{n-1}, \ldots$

Gen. series $f^{\operatorname{PreLie}}(t)=y$ which satisfies $y=t \exp (y)$

Dual operad PreLie! $=$ Perm

Chain-cplx see ref.

Properties binary, quadratic, Koszul.

Alternative The relation is $a s(x, y, z)=a s(x, z, y)$.

Relationship PreLie-alg $\rightarrow$ Lie-alg, $\quad[x, y]=x \circ y-y \circ x$

Dend-alg $\rightarrow$ PreLie-alg, $\quad x \circ y:=x \prec y-y \succ x$

Brace-alg $\rightarrow$ PreLie-alg, forgetful functor

Unit

$1 \circ x=x=x \circ 1$

Comment

This is right pre-Lie algebra, also called right-symmetric algebra, or Vinberg algebra. The opposite type is left symmetric.

First appeared in [Ger63, Vin63].

Ref.

[CL01] F. Chapoton, M. Livernet, Pre-Lie algebras and the rooted trees operad. Internat. Math. Res. Notices 2001, no. 8, 395-408. 
Name

Notation

Def. oper. $\quad x y$

sym.

rel.

Free alg.

rep. $\mathcal{P}(n)$

$\operatorname{dim} \mathcal{P}(n)$

Gen. series

Dual operad

Chain-cplx

Properties

Alternative

Relationship

$$
\begin{aligned}
& \text { Com-alg } \rightarrow \text { Perm-alg, } \\
& \text { NAP-alg } \rightarrow \text { Perm-alg, } \\
& \text { Perm-alg } \rightarrow \text { Dias-alg }
\end{aligned}
$$

Unit

no, unless it is a commutative algebra

Comment

Ref.

[Cha01] F. Chapoton, Un endofoncteur de la catégorie des opérades. Dialgebras \& related operads, 105-110, Lect. Notes in Math., 1763, Springer, 2001. 
Name

Notation

Def. oper. sym.

rel.

Free alg.

rep. $\mathcal{P}(n)$

$\operatorname{dim} \mathcal{P}(n)$

Gen. series

Dual operad

Chain-cplx

Properties

Alternative

Relationship

Unit

Comment

Ref .

\section{Dipterous algebra}

Dipt

$x * y, x \prec y$

$\left\{\begin{aligned}(x * y) * z & =x *(y * z) & & \text { (associativity) } \\ (x \prec y) \prec z & =x \prec(y * z) & & \text { (dipterous relation) }\end{aligned}\right.$

$\operatorname{Dipt}(V)=\bigoplus_{n \geq 1}\left(\mathbb{K}\left[P T_{n}\right] \oplus \mathbb{K}\left[P T_{n}\right]\right) \otimes V^{\otimes n}$, for $n=1$ the two copies of $P T_{1}$ are identified

$\operatorname{Dipt}(n)=\left(\mathbb{K}\left[P T_{n}\right] \oplus \mathbb{K}\left[P T_{n}\right]\right) \otimes \mathbb{K}\left[\mathbb{S}_{n}\right], n \geq 2$, Dipt $_{n}=\mathbb{K}\left[P T_{n}\right] \oplus \mathbb{K}\left[P T_{n}\right], n \geq 2$

$1,2 \times 2 !, 6 \times 3 !, 22 \times 4 !, 90 \times 5 !, 394 \times 6 !, 1806 \times 7 !, \ldots, 2 C_{n} \times n !, \ldots$ where $C_{n}$ is the Schröder number: $\sum_{n \geq 1} C_{n} t^{n}=\frac{1+t-\sqrt{1-6 t+t^{2}}}{4}$

$f^{\operatorname{Dipt}}(t)=\frac{1-t-\sqrt{1-6 t+t^{2}}}{2}$

Dipt!

Isomorphic to the total complex of a certain explicit bicomplex

binary, quadratic, ns, Koszul.

"diptère" in French

Dend-alg $\rightarrow$ Dipt-alg, $\quad x * y:=x \prec y+y \succ x$, a variation: replace the dipterous relation by $(x \prec y) * z+(x * y) \prec z=x \prec(y * z)+x *(y \prec z)$ to get Hoch-algebras, see [Ler11]. Same properties.

$1 \prec x=0, x \prec 1=x, \quad 1 * x=x=x * 1$.

dipterous $=2$-fold in greek (the free algebra has 2 planar trees copies)

[LR03] J.-L. Loday, M. Ronco, Algèbres de Hopf colibres, C.R.Acad. Sci. Paris t. 337, Ser. I (2003), 153 -158. 
Name

Notation

Def. oper. $\quad x \dashv y, x * y$

sym.

$$
\text { rel. } \quad\left\{\begin{aligned}
(x * y) * z & =x *(y * z), \\
(x \dashv y) \dashv z & =x \dashv(y * z), \\
(x * y) \dashv z & =0 \\
(x \dashv y) * z & =0 \\
0 & =x *(y \dashv z) \\
0 & =x \dashv(y \dashv z) .
\end{aligned}\right.
$$

Free alg. $\quad \operatorname{Dipt}^{!}(V)=\bar{T}(V) \oplus \bar{T}(V)$

rep. $\mathcal{P}(n) \quad \operatorname{Dipt}^{!}(n)=\mathbb{K}^{2} \otimes \mathbb{K}\left[\mathbb{S}_{n}\right]$, Dipt $_{n}^{!}=\mathbb{K}^{2}, n \geq 2$

$\operatorname{dim} \mathcal{P}(n) \quad 1,2 \times 2 !, 2 \times 3 !, 2 \times 4 !, 2 \times 5 !, 2 \times 6 !, 2 \times 7 !, \ldots, 2 \times n !, \ldots$

Gen. series $\quad f^{\operatorname{Dipt}^{!}}(t)=\frac{t+t^{2}}{1-t}$

Dual operad $\quad$ Dipt $! !=$ Dipt

Chain-cplx see ref.

Properties $\quad$ binary, quadratic, ns, Koszul.

Alternative

Relationship $A s$-alg $\rightarrow D i p t^{!}$-alg, $\quad x \dashv y:=x y=: x \vdash y$

Unit

No

Comment

Ref.

[LR03] J.-L. Loday, M. Ronco, Algèbres de Hopf colibres, C.R.Acad. Sci. Paris t. 337, Ser. I (2003), 153 -158. 
Name

Notation

Def. oper. sym.

rel.

\section{Two-associative algebra}

$2 a s$

$x * y, x \cdot y$,

$\left\{\begin{aligned}(x * y) * z & =x *(y * z) \\ (x \cdot y) \cdot z & =x \cdot(y \cdot z) .\end{aligned}\right.$

Free alg. $\quad 2 a s(V)=\bigoplus_{n \geq 1}\left(\mathbb{K}\left[T_{n}\right] \oplus \mathbb{K}\left[T_{n}\right]\right) \otimes V^{\otimes n}$, where $T_{n}=$ planar trees for $n=1$ the two copies of $T_{1}$ are identified

rep. $\mathcal{P}(n) \quad 2 a s(n)=\left(\mathbb{K}\left[T_{n}\right] \oplus \mathbb{K}\left[T_{n}\right]\right) \otimes \mathbb{K}\left[\mathbb{S}_{n}\right]$, so $\operatorname{Dipt}_{n}=\mathbb{K}\left[T_{n}\right] \oplus \mathbb{K}\left[T_{n}\right], n \geq 2$

$\operatorname{dim} \mathcal{P}(n) \quad 1,2 \times 2 !, 6 \times 3 !, 22 \times 4 !, 90 \times 5 !, 394 \times 6 !, 1806 \times 7 !, \ldots, 2 C_{n} \times n !, \ldots$ where $C_{n}$ is the Schröder number: $\sum_{n \geq 1} C_{n} t^{n}=\frac{1+t-\sqrt{1-6 t+t^{2}}}{4}$

Gen. series $f^{2 a s}(t)=\frac{1-t-\sqrt{1-6 t+t^{2}}}{2}$

Dual operad $2 a s^{!}$

Chain-cplx Isomorphic to the total complex of a certain explicit bicomplex

Properties $\quad$ binary, quadratic, ns, set-theoretic, Koszul.

Alternative

Relationship $2 a s$-alg $\rightarrow$ Dup-alg, $\quad 2 a s$-alg $\rightarrow B_{\infty}$-alg

Unit $\quad 1 \cdot x=x=x \cdot 1, \quad 1 * x=x=x * 1$.

Comment

Ref.

[LR06] J.-L. Loday, M. Ronco, On the structure of cofree Hopf algebras, J. reine angew. Math. 592 (2006), 123-155. 
Name

Notation

Def. oper. sym.

$$
\text { rel. } \quad\left\{\begin{aligned}
(x * y) * z & =x *(y * z), \\
(x \cdot y) \cdot z & =x \cdot(y \cdot z), \\
(x \cdot y) * z & =0 \\
(x * y) \cdot z & =0 \\
0 & =x *(y \cdot z) \\
0 & =x \cdot(y * z) .
\end{aligned}\right.
$$

Free alg. $\quad 2 a s^{!}(V)=V \oplus \oplus_{n \geq 2} V^{\otimes n} \oplus V^{\otimes n}$

rep. $\mathcal{P}(n) \quad \operatorname{Dipt}^{!}(n)=\mathbb{K}\left[\mathbb{S}_{n}\right] \oplus \mathbb{K}\left[\mathbb{S}_{n}\right], \quad \operatorname{Dipt}_{n}^{!}=\mathbb{K} \oplus \mathbb{K}, n \geq 2$.

$\operatorname{dim} \mathcal{P}(n) \quad 1,2 \times 2 !, 2 \times 3 !, 2 \times 4 !, 2 \times 5 !, 2 \times 6 !, 2 \times 7 !, \ldots, 2 \times n !, \ldots$

Gen. series $\quad f^{2 a s^{!}}(t)=\frac{t+t^{2}}{1-t}$

Dual operad $\left(2 a s^{!}\right)^{!}=2 a s$

Chain-cplx see ref.

Properties binary, quadratic, Koszul.

Alternative

Relationship

Unit

No

Comment

Ref .

[LR06] J.-L. Loday, M. Ronco, On the structure of cofree Hopf algebras, J. reine angew. Math. 592 (2006), 123-155. 
Name

Notation

Def. oper. sym.

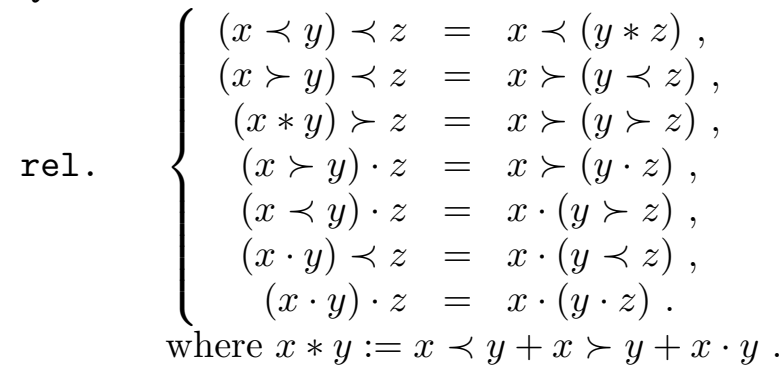

Free alg. Planar rooted trees with variables in between the leaves

rep. $\mathcal{P}(n) \quad$ Tridend $(n)=\mathbb{K}\left[P T_{n}\right] \otimes \mathbb{K}\left[\mathbb{S}_{n}\right]$, Tridend $_{n}=\mathbb{K}\left[P T_{n}\right]$

$\operatorname{dim} \mathcal{P}(n) \quad 1,3 \times 2 !, 11 \times 3 !, 45 \times 4 !, 197 \times 5 !, 903 \times 6 !, \ldots, C_{n} \times n !, \ldots$ where $C_{n}$ is the Schröder (or super Catalan) number

Gen. series

$f^{\text {Tridend }}(t)=\frac{-1+3 t+\sqrt{1-6 t+t^{2}}}{4 t}$

Dual operad Triend! $=$ Trias

Chain-cplx Isomorphic to the total complex of a certain explicit tricomplex

Properties binary, quadratic, ns, Koszul.

Alternative sometimes called dendriform trialgebras

Relationship Tridend-alg $\rightarrow$ As-alg, $\quad x * y:=x \prec y+y \succ x+x \cdot y$, ComTridend-alg $\rightarrow$ Tridend-alg, $\quad x \prec y:=x \cdot y=: y \succ x$

Unit $1 \prec x=0, x \prec 1=x, \quad 1 \succ x=x, x \succ 1=0, \quad 1 \cdot x=0=x \cdot 1$.

Comment There exist several variations (see [Cha02] for instance).

Ref.
[LR04] J.-L. Loday and M. Ronco, Trialgebras and families of polytopes, Contemporary Mathematics (AMS) 346 (2004), 369-398. 
Name

Notation

Def. oper. $\quad x \dashv y, x \vdash y, x \perp y \quad$ (no symmetry)

rel. $\quad\left\{\begin{array}{l}(x \dashv y) \dashv z=x \dashv(y \dashv z), \\ (x \dashv y) \dashv z=x \dashv(y \vdash z), \\ (x \vdash y) \dashv z=x \vdash(y \dashv z), \\ (x \dashv y) \vdash z=x \vdash(y \vdash z), \\ (x \vdash y) \vdash z=x \vdash(y \vdash z), \\ (x \dashv y) \dashv z=x \dashv(y \perp z), \\ (x \perp y) \dashv z=x \perp(y \dashv z), \\ (x \dashv y) \perp z=x \perp(y \vdash z), \\ (x \vdash y) \perp z=x \vdash(y \perp z), \\ (x \perp y) \vdash z=x \vdash(y \vdash z), \\ (x \perp y) \perp z=x \perp(y \perp z) .\end{array}\right.$

one relation for each cell of the pentagon.

Free alg. noncommutative polynomials with several variables marked

rep. $\mathcal{P}(n) \quad$ Trias $(n)=\mathbb{K}^{2^{n}-1} \otimes \mathbb{K}\left[\mathbb{S}_{n}\right], \quad$ Trias $_{n}=\mathbb{K}^{2^{n}-1}$

$\operatorname{dim} \mathcal{P}(n) \quad 1,3 \times 2 !, 7 \times 3 !, 15 \times 4 !, 31 \times 5 !, 63 \times 6 !, 127 \times 7 !, \ldots,\left(2^{n}-1\right) \times n !, \ldots$

Gen. series $\quad f^{\text {Trias }}(t)=\frac{t}{(1-t)(1-2 t)}$

Dual operad Trias $^{!}=$Tridend

Properties $\quad$ binary, quadratic, ns, set-theoretic, Koszul.

Alternative Also called associative trialgebra, or for short, trialgebra.

Relationship As-alg $\rightarrow$ Trias-alg, $\quad x \dashv y=x \vdash y=x \perp y=x y$

Unit $\quad$ Bar-unit: $x \dashv 1=x=1 \vdash x, 1 \dashv x=0=x \vdash 1,1 \perp x=0=x \perp 1$

Comment Relations easy to understand in terms of planar trees

Ref.
[LR04] J.-L. Loday and M. Ronco, Trialgebras and families of polytopes, Contemporary Mathematics (AMS) 346 (2004), 369-398. 
Name

Notation

Def. oper. $\quad x \circ y,[x, y]$

sym. $\quad[x, y]=-[y, x]$

rel. $\quad[x,[y, z]]+[y,[z, x]]+[z,[x, y]]=0$

$(x \circ y) \circ z-x \circ(y \circ z)-(x \circ z) \circ y+x \circ(z \circ y)=x \circ[y, z]$

$[x, y] \circ z=[x \circ z, y]+[x, y \circ z]$

Free alg. $\quad \operatorname{PostLie}(V) \cong \operatorname{Lie}(? ?(V))$

rep. $\mathcal{P}(n)$

$\operatorname{dim} \mathcal{P}(n)$

$1,3,20,210,3024, \cdots, a(n), \cdots$

Gen. series

$f(t)=-\log \left(\frac{1+\sqrt{1-4 t}}{2}\right)$

Dual operad

PostLie $e^{!}=$ComTrias

Chain-cplx

Properties

binary, quadratic, Koszul.

Alternative

Relationship PostLie-alg $\rightarrow$ ??-alg, $x y=x \circ y ? ? ?$

PostLie-alg $\rightarrow$ Lie-alg, $\{x, y\}=x \circ y-y \circ x+[x, y]$

PreLie-alg $\rightarrow$ PostLie-alg, $[x, y]=0$

Unit

Comment

Ref. [Val07] Vallette B., Homology of generalized partition posets, J. Pure Appl. Algebra 208 (2007), no. 2, 699-725. 
Name

Notation

Def . oper.

$$
\text { sym. }
$$$$
\text { rel. } \quad\left\{\begin{array}{l}
(x \dashv y) \dashv z=x \dashv(y \dashv z), \\
(x \dashv y) \dashv z=x \dashv(z \dashv y), \\
(x \dashv y) \dashv z=x \dashv(y \perp z), \\
(x \perp y) \dashv z=x \perp(y \dashv z), \\
(x \perp y) \perp z=x \perp(y \perp z) .
\end{array}\right.
$$

Free alg.

rep. $\mathcal{P}(n)$

$\operatorname{dim} \mathcal{P}(n)$

Gen. series $f(t)$

Dual operad ComTrias CostLie $^{\prime}=$ Post

Chain-cplx

Properties $\quad$ binary, quadratic, Koszul.

Alternative Triassociative with the following symmetry: $x \dashv y=y \vdash x$ and $x \perp y=y \perp x$

Relationship ComTrias-alg $\rightarrow$ Perm-alg

Unit

$x \dashv 1=x, 1 \dashv x=0,1 \perp x=0$

Comment

Ref. 
Name

Notation

Def. oper. $\quad x \prec y, x \cdot y$

sym. $\quad x \cdot y=y \cdot x$

rel. $\quad\left\{\begin{aligned}(x \prec y) \prec z & =x \prec(y \prec z)+x \prec(z \prec y), \\ (x \cdot y) \prec z & =x \cdot(y \prec z), \\ (x \prec z) \cdot y & =x \cdot(y \prec z), \\ (x \cdot y) \cdot z & =x \cdot(y \cdot z) .\end{aligned}\right.$

Free alg. $\quad C T D(V)=$ quasi-shuffle algebra on $V=Q \operatorname{Sym}(V)$

rep. $\mathcal{P}(n) \quad C T D(n)=$

$\operatorname{dim} \mathcal{P}(n) \quad 1,3,13,75,541,4683, \ldots$

Gen. series $\quad f^{C T D}(t)=\frac{\exp (t)-1}{2-\exp (t)}$

Dual operad $\quad C T D^{!}=$

Chain-cplx

Properties binary, quadratic, Koszul.

Alternative Handy to introduce $x * y:=x \prec y+y \prec x+x \cdot y$ (assoc. and comm.) equivalently: tridendriform with symmetry:

$x \prec y=y \succ x, x \cdot y=y \cdot x$

Relationship $C T D$-alg $\rightarrow$ Tridend-alg

Unit

$1 \prec x=0, x \prec 1=x, \quad 1 \cdot x=0=x \cdot 1$.

Comment

Ref.

[Lod07] J.-L. Loday, On the algebra of quasi-shuffles, Manuscripta mathematica 123 (1), (2007), 79-93. 
Name

Notation

Def . oper.

sym.

rel.

\section{Dual CTD algebra}

$C T D^{!}$

$x \dashv y,[x, y]$

$[x, y]=-[y, x]$

Free alg.

rep. $\mathcal{P}(n)$

$\operatorname{dim} \mathcal{P}(n) \quad 1,3, \ldots$

Gen. series $\quad f^{C T D^{!}}(t)=$

Dual operad $\left(C T D^{!}\right)^{!}=C T D$

Chain-cplx

Properties $\quad$ binary, quadratic, Koszul.

Alternative

Relationship Trias-alg $\rightarrow C T D^{!}$-alg

Unit

Comment

Ref. 
Name

Notation

Def. oper. sym. rel.

\section{Gerstenhaber algebra}

underlying objects: graded vector spaces

Gerst

$m=$ binary operation of degree $0, c=$ binary operation of degree 1 $m$ symmetric, $c$ antisymmetric

$c \circ_{1} c+\left(c \circ_{1} c\right)^{(123)}+\left(c \circ_{1} c\right)^{(321)}=0$, $c \circ_{1} m-m \circ_{2} c-\left(m \circ_{1} c\right)^{(23)}=0$, $m \circ_{1} m-m \circ_{2} m=0$.

Free alg.

rep. $\mathcal{P}(n)$

$\operatorname{dim} \mathcal{P}(n)$

Gen. series

$f(t)=$

Dual operad

Chain-cplx

Properties binary, quadratic, Koszul.

Alternative

Relationship

Unit

Comment

To get the relations in terms of elements, do not forget to apply the Koszul sign rule. Observe that the last relation is associativity.

Ref. 
Name

Notation

Def. oper. sym. rel.

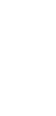

\section{Batalin-Vilkovisky algebra}

underlying objects: graded vector spaces

$$
B V \text {-alg }
$$

$\Delta$ unary degree $1, m$ binary degree $0, c$ binary degree 1 $m$ symmetric, $c$ antisymmetric

$m \circ_{1} m-m \circ_{2} m=0$,

$\Delta^{2}=0$,

$c=\Delta \circ_{1} m+m \circ_{1} \Delta+m \circ_{2} \Delta$,

$c \circ_{1} c+\left(c \circ_{1} c\right)^{(123)}+\left(c \circ_{1} c\right)^{(321)}=0$,

$c \circ_{1} m-m \circ_{2} c-\left(m \circ_{1} c\right)^{(23)}=0$,

$\Delta \circ_{1} c+c \circ_{1} \Delta+c \circ_{2} \Delta=0$.

Free alg.

rep. $\mathcal{P}(n)$

$\operatorname{dim} \mathcal{P}(n)$

Gen. series

$f(t)=$

Dual operad

Chain-cplx

Properties

unary and binary, inhomogeneous quadratic, Koszul.

Alternative

Generated by $\Delta$ and $m$ only.

Relationship

$B V$-alg $\rightarrow$ Gerst-alg

Unit

Comment

Defined by J.-L. Koszul. To get the relations in terms of elements, do not forget to apply the Koszul sign rule.

Ref . [GCTV09] 
Name

Notation

Def. oper.

sym.

rel.

\section{Magmatic algebra}

Mag

$x y$

any parenthesizing of words

rep. $\mathcal{P}(n)$

$\operatorname{Mag}(n)=\mathbb{K}\left[P B T_{n}\right] \otimes \mathbb{K}\left[\mathbb{S}_{n}\right], \quad M a g_{n}=\mathbb{K}\left[P B T_{n}\right]$ (planar binary trees with $n$ leaves)

$\operatorname{dim} \mathcal{P}(n) \quad 1,1 \times 2 !, 2 \times 3 !, 5 \times 4 !, 14 \times 5 !, 42 \times 6 !, 132 \times 7 !, \ldots, c_{n-1} \times n !, \ldots$ where $c_{n}=\frac{1}{n+1}\left(\begin{array}{c}2 n \\ n\end{array}\right)$ (Catalan number)

Gen. series $\quad f^{M a g}(t)=(1 / 2)(1-\sqrt{1-4 t})$

Dual operad $M a g^{!}=N i l_{2}$

Chain-cplx

Properties $\quad$ binary, quadratic, ns, set-theoretic, Koszul.

Alternative sometimes (improperly) called nonassociative algebra.

Relationship many "inclusions" (all types of alg. with only one gen. op.)

Unit $\quad 1 x=x=x 1$

Comment

Ref. 


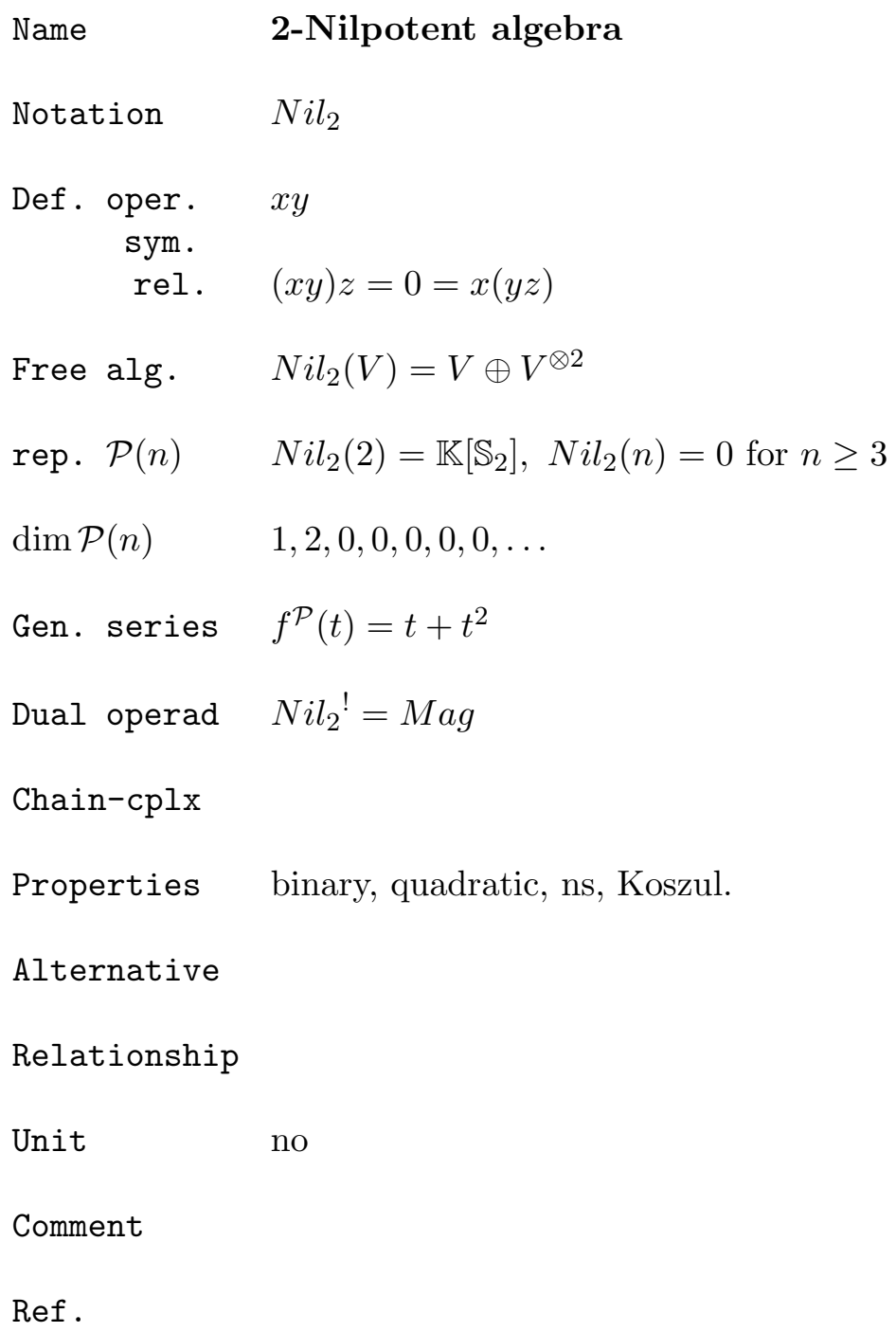


Name

Notation

Def. oper. sym.

rel.

\section{Commutative magmatic algebra}

ComMag

Free alg.

any parenthesizing of commutative words

rep. $\mathcal{P}(n)$

$\operatorname{ComMag}(n)=\mathbb{K}[\operatorname{shBT}(n)]$ $\operatorname{sh} B T(n)=\{$ shuffle binary trees with $n$ leaves $\}$

$\operatorname{dim} \mathcal{P}(n)$ $\operatorname{dim} \operatorname{ComMag}(n)=(2 n-3) ! !=1 \times 3 \times \cdots \times(2 n-3)$

Gen. series $f^{\operatorname{ComMag}}(t)=$

Dual operad

ComMag!-alg: $[x, y]$ antisymmetric, $[[x, y], z]=0$.

Chain-cplx

Properties binary, quadratic, set-theoretic, Koszul.

Alternative

Relationship ComMag $\longmapsto$ PreLie, $x \cdot y:=\{x, y\}+\{y, x\}$, cf. [BL11]

Unit $\quad 1 x=x=x 1$

Comment

Ref. 
Name

Notation

Def . oper.

sym.

rel.

Free alg.

rep. $\mathcal{P}(n)$

$\operatorname{dim} \mathcal{P}(n)$

Gen. series

Dual operad

Chain-cplx

Properties

Alternative

Relationship

Unit

Comment

Ref.

\section{Anti-symmetric nilpotent algebra}

ComMag!

$x \cdot y$

$x \cdot y=-y \cdot x$

$(x y) z=0$

ComMag.

binary, quadratic, set-theoretic, Koszul. 


\section{Name Quadri-algebra}

Notation Quadri

Def. oper. $\quad x \nwarrow y, x \nearrow y, x \searrow y, x \swarrow y \quad$ called NW, NE, SE, SW oper. rel.

$$
\begin{aligned}
& (x \nwarrow y) \nwarrow z=x \nwarrow(y \star z) \quad(x \nearrow y) \nwarrow z=x \nearrow(y \prec z) \quad(x \wedge y) \nearrow z=x \nearrow(y \succ z) \\
& (x \swarrow y) \nwarrow z=x \swarrow(y \wedge z) \quad(x \searrow y) \nwarrow z=x \searrow(y \nwarrow z) \quad(x \vee y) \nearrow z=x \searrow(y \nearrow z) \\
& (x \prec y) \swarrow z=x \swarrow(y \vee z) \quad(x \succ y) \swarrow z=x \searrow(y \swarrow z) \quad(x \star y) \searrow z=x \searrow(y \searrow z)
\end{aligned}
$$

where

$$
\begin{aligned}
x \succ y & :=x \nearrow y+x \searrow y, \quad x \prec y:=x \nwarrow y+x \swarrow y \\
x \vee y & :=x \searrow y+x \swarrow y, \quad x \wedge y:=x \nearrow y+x \nwarrow y \\
x \star y & :=x \searrow y+x \nearrow y+x \nwarrow y+x \swarrow y \\
& =x \succ y+x \prec y=x \vee y+x \wedge y
\end{aligned}
$$

Free alg.

rep. $\mathcal{P}(n)$

$\operatorname{dim} \mathcal{P}(n) \quad 1,4 \times 2 !, 23 \times 3 !, 156 \times 4 !, 1162 \times 5 !, 9192 \times 6 !, \ldots$

Gen. series $f(t)$

$\operatorname{dim} \mathcal{P}_{n}=\frac{1}{n} \sum_{j=n}^{2 n-1}\left(\begin{array}{c}3 n \\ n+1+j\end{array}\right)\left(\begin{array}{c}j-1 \\ j-n\end{array}\right)$

Dual operad Quadri!

Properties binary, quadratic, ns, Koszul.

Alternative $\quad$ Quadri $=$ Dend $\mathbf{\square}$ Dend $=$ PreLie $\bullet$ Dend

Relationship Related to dendriform in several ways

Unit partial unit (like in dendriform)

Comment There exist several variations like $\mathcal{P} \mathbf{D}$ (cf. Ph. Leroux [Ler04])

Ref . $\quad$ [AL04] M. Aguiar, J.-L. Loday, Quadri-algebras,

J. Pure Applied Algebra 191 (2004), 205-221. 
Name

Notation

Def. oper. $\quad x \nwarrow y, x \nearrow y, x \searrow y, x \swarrow y$

sym.

rel. A FAIRE

Free alg.

rep. $\mathcal{P}(n)$

$\operatorname{dim} \mathcal{P}(n)$

Gen. series

Dual operad

Chain-cplx

Properties

Alternative

Relationship

Unit

Comment

Ref .

[Val08] B.Vallette, Manin products, Koszul duality, Loday algebras and Deligne conjecture.

J. Reine Angew. Math. 620 (2008), 105-164. 
Name

Notation

Def. oper. sym.

rel. $\quad \begin{cases}(x \prec y) \prec z= & x \prec(y \prec z), \\ (x \succ y) \prec z= & x \succ(y \prec z), \\ (x \succ y) \succ z= & x \succ(y \succ z) .\end{cases}$

Free alg. $\quad \operatorname{Dup}(V)=\oplus_{n \geq 1} \mathbb{K}\left[P B T_{n+1}\right] \otimes V^{\otimes n}$, for p.b. trees $s$ and $t$ : $x \succ y$ over operation is grafting of $x$ on the leftmost leaf of $y$ $x \prec y$ under operation is grafting of $y$ on the rightmost leaf of $x$

rep. $\mathcal{P}(n) \quad \operatorname{Dup}(n)=\mathbb{K}\left[P B T_{n+1}\right] \otimes \mathbb{K}\left[\mathbb{S}_{n}\right]$, so $\operatorname{Dup} p_{n}=\mathbb{K}\left[P B T_{n+1}\right]$

$\operatorname{dim} \mathcal{P}(n)$

Gen. series

$1,2 \times 2 !, 5 \times 3 !, 14 \times 4 !, 42 \times 5 !, 132 \times 6 !, 429 \times 7 !, \ldots, c_{n} \times n !, \ldots$ where $c_{n}=\frac{1}{n+1}\left(\begin{array}{c}2 n \\ n\end{array}\right)$ is the Catalan number

Dual operad

Chain-cplx Isomorphic to the total complex of a certain explicit bicomplex

Properties $\quad$ binary, quadratic, ns, set-theoretic, Koszul.

Alternative

Relationship $2 a s$-alg $\rightarrow$ Dup-alg $\rightarrow A s^{2}$-alg

Unit

Comment

The associator of $x y:=x \succ y-x \prec y$ is $a s(x, y, z)=x \prec(y \succ z)-(x \prec y) \succ z$. Appeared first in [BF03]

Ref . Astérisque (2008), no 320, x+116 p. 
Name

\section{Dual duplicial algebra}

Notation

Dup!

Def. oper.

$x \prec y, x \succ y$

sym.

rel. $\quad\left\{\begin{aligned}(x \prec y) \prec z & =x \prec(y \prec z), \\ (x \prec y) \succ z & =0, \\ (x \succ y) \prec z & =x \succ(y \prec z), \\ 0 & =x \prec(y \succ z), \\ (x \succ y) \succ z & =x \succ(y \succ z) .\end{aligned}\right.$

Free alg. $\quad \operatorname{Dup}^{!}(V)=\oplus_{n \geq 1} n V^{\otimes n}$

noncommutative polynomials with one variable marked

rep. $\mathcal{P}(n) \quad D u p !(n)=\mathbb{K}^{n} \otimes \mathbb{K}\left[\mathbb{S}_{n}\right], \quad$ Dup! ${ }_{n}=\mathbb{K}^{n}$

$\operatorname{dim} \mathcal{P}(n) \quad 1,2 \times 2 !, 3 \times 3 !, 4 \times 4 !, 5 \times 5 !, 6 \times 6 !, 7 \times 7 !, \ldots, n \times n !, \ldots$,

Gen. series $\quad f^{D u p^{!}}(t)=\frac{t}{(1-t)^{2}}$

Dual operad $\quad$ Dup $! !=$ Dup

Chain-cplx see ref.

Properties binary, quadratic, Koszul.

Alternative

Relationship

Unit

Comment

Ref. 


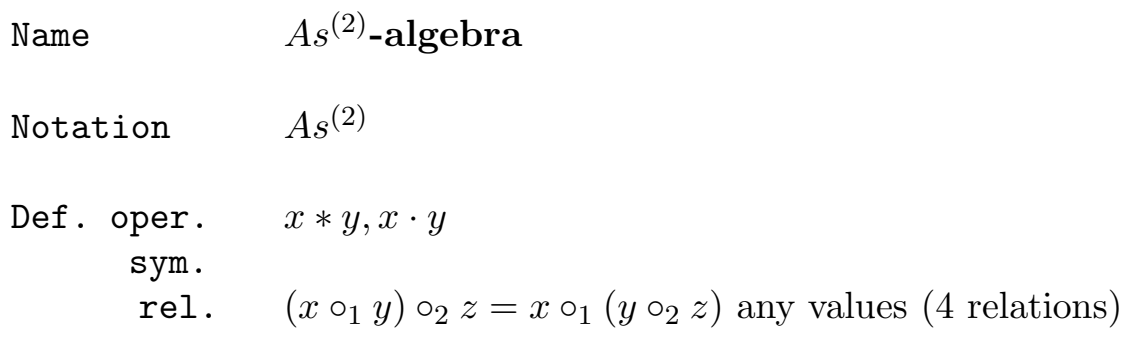

Ref. 
Name

Notation

Def. oper.

sym.

rel.
$A s^{\langle 2\rangle}$-algebra

$A s^{\langle 2\rangle}$, compatible products algebra

$x * y, x \cdot y$

$(x * y) * z=x *(y * z)$

$(x * y) \cdot z+(x \cdot y) * z=x *(y \cdot z)+x \cdot(y * z)$

$(x \cdot y) \cdot z=x \cdot(y \cdot z)$

Free alg. $\quad$ similar to dendriform

rep. $\mathcal{P}_{n} \quad A s_{n}^{\langle 2\rangle}=\mathbb{K}\left[P B T_{n+1}\right]$

$\operatorname{dim} \mathcal{P}_{n}$

$c_{n}=\frac{1}{n+1}\left(\begin{array}{c}2 n \\ n\end{array}\right)$

Gen. series

$f^{A s^{\langle 2\rangle}}(t)=\frac{1-2 t-\sqrt{1-4 t}}{2 t}$

Dual operad

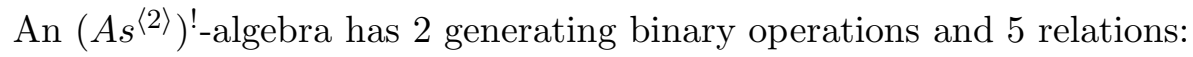
$\left(x \circ_{i} y\right) \circ_{j} z=x \circ_{i}\left(y \circ_{j} z\right)$ and $(x * y) \cdot z+(x \cdot y) * z=x *(y \cdot z)+x \cdot(y * z)$

Chain-cplx

Properties binary, quadratic, ns, Koszul.

Alternative

Relationship $\left(A s^{\langle 2\rangle}\right)$ !-alg $\rightarrow A s^{(2)}$-alg $\rightarrow A s^{\langle 2\rangle}$-alg

Unit

Comment equivalently $\lambda x * y+\mu x \cdot y$ is associative for any $\lambda, \mu$ Variations: $A s^{\langle k\rangle}$, Hoch-alg.

Ref. 
Name

Notation

Def. oper. sym.

rel.

\section{L-dendriform algebra}

$L$-dend

$x \triangleright y$ and $x \triangleleft y$

$x \triangleright(y \triangleright z)-(x \bullet y) \triangleright z=y \triangleright(x \triangleright z)-(y \bullet x) \triangleright z$ $x \triangleright(y \triangleleft z)-(x \triangleright y) \triangleleft z=y \triangleleft(x \bullet z)-(y \triangleleft x) \triangleleft z$ where $x \bullet y:=x \triangleright y+x \triangleleft y$.

Free alg.

rep. $\mathcal{P}(n)$

$\operatorname{dim} \mathcal{P}(n)$

Gen. series $f(t)=$

Dual operad

Chain-cplx

Properties

L-dend $=$ PreLie $\bullet$ PreLie ?

Alternative

Relationship Dend-alg $\rightarrow L$-dend-alg via $x \triangleright y:=x \succ y$ and $x \triangleleft y:=x \prec y$ L-dend-alg $\rightarrow$ PreLie-alg, $(A, \triangleright, \triangleleft) \mapsto(A, \bullet)$

Unit

Comment

Various variations like $L$-quad-alg [Bai10]

Ref.

Bai C.M., Liu L.G., Ni X., L-dendriform algebras, preprint (2010). 
Name

Notation

Def . oper.

sym.

rel.

\section{Lie-admissible algebra}

Lie-adm

$x y$

$[x, y]=x y-y x$ is a Lie bracket, that is $\sum_{\sigma} \operatorname{sgn}(\sigma) \sigma((x y) z-x(y z))=0$

Free alg.

rep. $\mathcal{P}(n) \quad \operatorname{Lie}-a d m(n)=$ ?

$\operatorname{dim} \mathcal{P}(n) \quad 1,2,11, \ldots$

Gen. series $f^{\text {Lie-adm }}(t)=$ ? its dimensions are $1,2,1, ? ?$

Dual operad Lie-adm!

Chain-cplx

Properties binary, quadratic, Koszul ??.

Alternative

Relationship $A s$-alg $\rightarrow$ PreLie-alg $\rightarrow$ Lie-adm-alg

Unit

Comment

Ref. 
Name

Notation

Def. oper. sym.

Free alg.

$$
\text { rel. } \quad\left\{\begin{aligned}
(x \prec y) \prec z & =x \prec(y * z), \\
(x \prec y) \prec z & =x \prec(z * y), \\
(x * y) \succ z & =x \succ(y \succ z), \\
(x \succ y) \prec z & =x \succ(z \succ y), \\
(x \succ y) \prec z & =x \succ(y \prec z),
\end{aligned}\right.
$$

rep. $\mathcal{P}(n)$

$\operatorname{dim} \mathcal{P}(n) \quad 1,6$,

Gen. series

Dual operad PermPreLie $=$ Perm $\circ$ PreLie $=$ Perm $\otimes$ PreLie

Chain-cplx

Properties binary, quadratic, set-theoretic.

Alternative

Relationship PreLiePerm = PreLie $\bullet$ Perm, see [Val08] Zinb-alg $\rightarrow$ PreLiePerm-alg $\rightarrow$ Dend-alg

Unit

no

Comment

Ref.

[Val08] B.Vallette, Manin products, Koszul duality, Loday algebras and Deligne conjecture.

J. Reine Angew. Math. 620 (2008), 105-164. 
Name

Notation

Def . oper. sym.

rel.

\section{Alternative algebra}

Altern

$x y$

$(x y) z-x(y z)=-(y x) z+y(x z)$,

$(x y) z-x(y z)=-(x z) y+x(z y)$,

Free alg.

rep. $\mathcal{P}(n)$

$\operatorname{dim} \mathcal{P}(n)$

$1,2,7,32,175, ? ?$

Gen. series

$f(t)$

Dual operad $(x y) z=x(y z)$,

$x y z+y x z+z x y+x z y+y z x+z y x=0$

$\operatorname{dim}$ Altern $^{!}(n)=1,2,5,12,15, \ldots$

Chain-cplx

Properties binary, quadratic, nonKoszul [DZ09].

Alternative Equivalent presentation: the associator as $(x, y, z)$

is skew-symmetric: $\sigma \cdot a s(x, y, z)=\operatorname{sgn}(\sigma) a s(x, y, z)$

Relationship As-alg $\rightarrow$ Altern-alg

Unit $\quad 1 x=x=x 1$

Comment The octonions are an example of alternative algebra Integration: Moufang loops $\operatorname{dim} \mathcal{P}(n)$ computed by W. Moreira

Ref .

[She04] Shestakov, I. P., Moufang loops and alternative algebras.

Proc. Amer. Math. Soc. 132 (2004), no. 2, 313-316. 
Name

Notation

Def. oper.

sym.

rel.

\section{Parametrized-one-relation algebra}

\section{Param1rel}

$x y$

none

$(x y) z=\sum_{\sigma \in \mathbb{S}_{3}} a_{\sigma} \sigma \cdot x(y z)$ where $a_{\sigma} \in \mathbb{K}$

Free alg.

rep. $\mathcal{P}(n)$

$\operatorname{dim} \mathcal{P}(n)$

Gen. series $f(t)=$

Dual operad $\quad x(y z)=\sum_{\sigma \in \mathbb{S}_{3}} \operatorname{sgn}(\sigma) a_{\sigma} \sigma^{-1} \cdot(x y) z$

Chain-cplx

Properties

Alternative

Relationship Many classical examples are particular case: As, Leib, Zinb, Pois

Unit

Comment

Problem: for which families of parameters $\left\{a_{\sigma}\right\}_{\sigma \in \mathbb{S}_{3}}$ is the operad a Koszul operad?

Ref. 
Def. oper.

$\left(x_{1}, \ldots, x_{n}\right)_{i}^{n}$ for $1 \leq i \leq n-2, n \geq 3$

sym.

rel.

Free alg. Described in terms of some coloured planar rooted trees

rep. $\mathcal{P}(n) \quad$ a sum of regular representations indexed as said above

$\operatorname{dim} \mathcal{P}(n) \quad 1,0,1 \times 3 !, 2 \times 4 !, 6 \times 5 !, 18 \times 6 !, 57 \times 7 !, \ldots, F_{n-1} \times n !, \ldots$ where $F_{n}=$ Fine number

Gen. series

$f^{\text {MagFine }}(t)=\frac{1+2 t-\sqrt{1-4 t}}{2(2+t)}$

Dual operad

MagFine! same generating operations, any composition is trivial $\operatorname{dim}$ MagFine $e_{n}^{!}=n-2$

$f^{\text {MagFine! }}(t)=t+\frac{t^{3}}{(1-t)^{2}}$

Chain-cplx

Properties multi-ary, quadratic, ns, Koszul.
Alternative

Relationship

Unit

Comment

Ref .
[HLR08] Holtkamp, R., Loday, J.-L., Ronco, M., Coassociative magmatic bialgebras and the Fine numbers, J.Alg.Comb. 28 (2008), 97-114. 
Name

Notation

Def . oper. sym.

rel.

\section{Generic magmatic algebra}

\section{GenMag}

$a_{n}$ generating operations of arity $n, a_{1}=1$

Free alg.

rep. $\mathcal{P}(n)$

a sum of regular representations

$\operatorname{dim} \mathcal{P}(n)$

Gen. series

$f^{\operatorname{GenMag}}(t)=\sum_{n} b_{n} t^{n}, b_{n}=$ polynomial in $a_{1}, \cdots, a_{n}$

Dual operad

same generating operations, any composition is trivial $\operatorname{dim} \operatorname{GenMag} g_{n}^{!}=a_{n}, f^{\text {GenMag }}(t)=\sum_{n} a_{n} t^{n}$

Chain-cplx

Properties multi-ary, quadratic, ns, Koszul.

Alternative

Relationship For MagFine $a_{n}=n-2$.

Unit

Comment

give a nice proof of the inversion formula for a generic power series (computation of the polynomial $b_{n}$ )

Ref .

[Lod05] Loday, J.-L., Inversion of integral series enumerating planar trees. Séminaire lotharingien Comb. 53 (2005), exposé B53d, 16pp. 
Name Nonassociative permutative algebra

Notation $\quad$ NAP

Def. oper. $\quad x y$

sym.

rel. $(x y) z=(x z) y$

Free alg. $\quad N A P(V)$ can be described in terms of rooted trees

rep. $\mathcal{P}(n) \quad N A P(n)=\operatorname{PreLie}(n)$ as $\mathbb{S}_{n}$-modules

$\operatorname{dim} \mathcal{P}(n) \quad 1,2,9,64,625,1296,117649, \ldots, n^{n-1}, \ldots$

Gen. series $f^{N A P}(t)=y$ which satisfies $y=t \exp (y)$

Dual operad $N A P^{!}$

Chain-cplx

Properties binary, quadratic, set-theoretic, Koszul.

Alternative

Relationship Perm-alg $\rightarrow N A P$-alg

Unit no

Comment This is right NAP algebra

Ref. [Liv06] Livernet, M., A rigidity theorem for pre-Lie algebras, J. Pure Appl. Algebra 207 (2006), no. 1, 1-18. 
Name

Notation

Def . oper. sym.

rel.

$x(y z)+z(y x)=(x y) z+(z y) x$

$((x y) z) t+((z y) x) t=x(y(z t))+z(y(x t))$, $t(x(y z)+z(y x))=((t x) y) z+((t z) y) x$, $(x y)(t z)+(z y)(t x)=(x(y t)) z+(z(y t)) x$

Free alg.

rep. $\mathcal{P}(n)$

$\operatorname{dim} \mathcal{P}(n)$

$1,2,7,40, ? ?$

Gen. series

$f(t)=$

Dual operad

Chain-cplx

Properties binary

Alternative Relation sometimes written in terms of the Jacobiator

Relationship Altern-alg $\stackrel{-}{\longrightarrow}$ Moufang-alg $\rightarrow$ NCJordan-alg

Unit

Comment Integration: Moufang loop.

From this presentation there is an obvious definition of "nonantisymmetric Malcev algebra".

Ref . [PIS04] Shestakov, I., Pérez-Izquierdo, J.M., An envelope for Malcev algebras. J. Alg. 272 (2004), 379-393. 
Name

Notation

Def . oper. sym. rel.
Malcev algebra

Malcev

$x y$

$x y=-y x$

$((x y) z) t+(x(y z)) t+x((y z) t)+x(y(z t))=(x y)(z t)$

Free alg.

rep. $\mathcal{P}(n)$

$\operatorname{dim} \mathcal{P}(n) \quad 1,1,3,9, ? ?$

Gen. series $f(t)$

Dual operad

Chain-cplx

Properties cubic.

Alternative

Relationship Altern-alg $\longrightarrow$ Malcev-alg, Lie-alg $\rightarrow$ Malcev-alg

Unit

Comment

Ref . [PIS04] Shestakov, I., Pérez-Izquierdo, J.M., An envelope for Malcev algebras. J. Alg. 272 (2004), 379-393. 
Name

Notation

Def. oper.

sym.

rel.

\section{Novikov algebra}

Novikov

$x y$

$(x y) z-x(y z)=(x z) y-x(z y)$ $x(y z)=y(x z)$

Free alg.

rep. $\mathcal{P}(n)$

$\operatorname{dim} \mathcal{P}(n) \quad 1,2$,

Gen. series $f^{\text {Novikov }}(t)=$

Dual operad

Chain-cplx

Properties binary, quadratic.

Alternative Novikov is pre-Lie $+x(y z)=y(x z)$

Relationship Novikov-alg $\rightarrow$ PreLie-alg

Unit

Comment

Ref. 
Name

Notation

Def. oper. $\quad[x, y],\{x, y\}$

sym. $\quad[x, y]=-[y, x],\{x, y\}=\{y, x\}$

rel. Any linear combination is a Lie bracket

Free alg.

rep. $\mathcal{P}(n)$

$\operatorname{dim} \mathcal{P}(n)$

Gen. series $f(t)=$

Dual operad

Chain-cplx

Properties binary, quadratic.

Alternative

Relationship

Unit

Comment

Ref .

[DK07] Dotsenko V., Khoroshkin A., Character formulas for the operad of two compatible brackets and for the bihamiltonian operad, Functional Analysis and Its Applications, 41 (2007), no.1, 1-17. 
Name

Notation

Def . oper. sym. rel.

\section{DipreLie algebra}

DipreLie

$x \circ y, x \bullet y$

$(x \circ y) \circ z-x \circ(y \circ z)=(x \circ z) \circ y-x \circ(z \circ y)$

$(x \bullet y) \bullet z-x \bullet(y \bullet z)=(x \bullet z) \bullet y-x \bullet(z \bullet y)$

$(x \circ y) \bullet z-x \circ(y \bullet z)=(x \bullet z) \circ y-x \bullet(z \circ y)$

Free alg.

rep. $\mathcal{P}(n)$

$\operatorname{dim} \mathcal{P}(n)$

Gen. series $f(t)=$

Dual operad

Chain-cplx

Properties binary, quadratic.

Alternative

Relationship relationship with the Jacobian conjecture (T. Maszczsyk)

Unit

Comment

Ref. Maszczsyk T., unpublished. 
Name

Notation

Def . oper. sym. rel.

\section{Akivis algebra}

Akivis

$[x, y],(x, y, z)$

$[x, y]=-[y, x]$

$[[x, y], z]+[[y, z], x]+[[z, x], y]=$ $(x, y, z)+(y, z, x)+(z, x, y)-(x, z, y)-(y, x, z)-(z, y, x)$

(Akivis relation)

Free alg.

rep. $\mathcal{P}(n)$

$\operatorname{dim} \mathcal{P}(n)$

$1,1,8, \ldots$

Gen. series

$f(t)=$

Dual operad

Chain-cplx

Properties binary and ternary, quadratic.

Alternative relation also called "nonassociative Jacobi identity"

Relationship Akivis-alg $\rightarrow$ Sabinin-alg, Mag-alg $\rightarrow$ Akivis-alg, $\quad[x, y]=x y-y x$, $(x, y, z)=(x y) z-x(y z)$

Unit

Comment

Ref.

[BHP05] 
Name

Notation

Def. oper.

sym.

rel.

\section{Free alg.}

rep. $\mathcal{P}(n)$

$\operatorname{dim} \mathcal{P}(n)$

Gen. series

Dual operad

Chain-cplx

Properties

Alternative

Relationship

Unit

Ref.

Comment Integration: local analytic loop

\section{Sabinin algebra}

\section{Sabinin}

$\left\langle x_{1}, \ldots, x_{m} ; y, z\right\rangle, m \geq 0$

$\Phi\left(x_{1}, \ldots, x_{m} ; y_{1}, \ldots, y_{n}\right), \quad m \geq 1, n \geq 2$,

$\left\langle x_{1}, \ldots, x_{m} ; y, z\right\rangle=-\left\langle x_{1}, \ldots, x_{m} ; z, y\right\rangle$

$\Phi\left(x_{1}, \ldots, x_{m} ; y_{1}, \ldots, y_{n}\right)=\Phi\left(\omega\left(x_{1}, \ldots, x_{m}\right) ; \theta\left(y_{1}, \ldots, y_{n}\right)\right), \omega \in \mathbb{S}_{m}, \theta \in \mathbb{S}_{n}$

$\left\langle x_{1}, \ldots, x_{r}, u, v, x_{r+1}, \ldots, x_{m} ; y, z\right\rangle-\left\langle x_{1}, \ldots, x_{r}, v, u, x_{r+1}, \ldots, x_{m} ; y, z\right\rangle$

$+\sum_{k=0}^{r} \sum_{\sigma}\left\langle x_{\sigma(1)}, \ldots, x_{\sigma(k)} ;\left\langle x_{\sigma(k+1)}, \ldots, x_{\sigma(r)} ; u, v\right\rangle, x_{r+1}, \ldots, x_{m} ; y, z\right\rangle$

where $\sigma$ is a $(k, r-k)$-shuffle

$K_{u, v, w}\left[\left\langle x_{1}, \ldots, x_{r} ; y, z\right\rangle+\right.$

$\left.\sum_{k=0}^{r} \sum_{\sigma}\left\langle x_{\sigma(1)}, \ldots, x_{\sigma(k)} ;\left\langle x_{\sigma(k+1)}, \ldots, x_{\sigma(r)} ; v, w\right\rangle, u\right\rangle\right]=0$

where $K_{u, v, w}$ is the sum over all cyclic permutations

quadratic, ns.

There exists a more compact form of the relations which uses the tensor algebra over the Sabinin algebra

Mag-alg $\longrightarrow$ Sabinin-alg, $\langle y, z\rangle=y z-z y,\langle x ; y, z\rangle=? ?$

Akivis-alg $\longrightarrow$ Sabinin-alg, $\langle y, z\rangle=-[y, z]$,

$\langle x ; y, z\rangle=(x, z, y)-(x, y, z),\left\langle x_{1}, \ldots, x_{m} ; y, z\right\rangle=0, m \geq 2$

[PI07] D. Pérez-Izquierdo, Algebras, hyperalgebras, nonassociative bialgebras and loops. Adv. in Maths 208 (2007), 834-876. 
Name

Notation

Def . oper.

sym.

rel.

\section{Jordan triples}

JT

$(x y z)$ or $(x, y, z)$

$(x y z)=(z y x)$

$(x y(z t u))=((x y z) t u)-(z(t x y) u)+(z t(x y u))$

Free alg.

rep. $\mathcal{P}(2 n-1)$

$\operatorname{dim} \mathcal{P}(2 n-1) \quad 1,3,50, ? ?$

Gen. series $f(t)$

Dual operad

Chain-cplx

Properties ternary, quadratic, Koszul ?.

Alternative

Relationship

Unit

Comment

Remark that the quadratic relation, as written here, has a Leibniz flavor. Dimension computed by Walter Moreira

Ref . 
Name

Notation

Def. oper.

sym.

rel.

\section{Totally associative ternary algebra}

$t-A s^{\langle 3\rangle}$

$(x y z)$

$((x y z) u v)=(x(y z u) v)=(x y(z u v))$

Free alg.

rep. $\mathcal{P}(2 n-1)$

$\operatorname{dim} \mathcal{P}(2 n-1)$

$1,3 !, 5 !, \ldots,(2 n+1) !, \ldots$

Gen. series

$f^{t-A s^{3}}(t)=\frac{t}{1-t^{2}}$

Dual operad

$t-A s^{\langle 3\rangle !}=p-A s^{\langle 3\rangle}$

Chain-cplx

Properties ternary, quadratic, ns, set-theoretic, Koszul ?.

Alternative

Relationship $\quad A s$-alg $\rightarrow t-A s^{\langle 3\rangle}$

Unit

Comment

Ref .

[Gne97] Gnedbaye, A.V., Opérades des algèbres $(k+1)$-aires. Operads: Proceedings of Renaissance Conferences, 83-113, Contemp. Math., 202, Amer. Math. Soc., Providence, RI, 1997. 
Name

Notation

Def. oper.

sym.

rel.
Partially associative ternary algebra

$p-A s^{\langle 3\rangle}$

$(x y z)$

$((x y z) u v)+(x(y z u) v)+(x y(z u v))=0$

Free alg.

rep. $\mathcal{P}(2 n-1)$

$\operatorname{dim} \mathcal{P}(2 n-1)$

Gen. series $f(t)$

Dual operad $\quad p-A s^{\langle 3\rangle !}=t-A s^{\langle 3\rangle}$

Chain-cplx

Properties $\quad$ ternary, quadratic, ns, Koszul ?.

Alternative

Relationship

Unit

Comment

Ref .

[Gne97] Gnedbaye, A.V., Opérades des algèbres $(k+1)$-aires. Operads: Proceedings of Renaissance Conferences, 83-113, Contemp. Math., 202, Amer. Math. Soc., Providence, RI, 1997. 
Name

Notation

Def. oper. $\quad[x y z]$

sym. $\quad[x y z]=-[y x z]$

$[x y z]+[y z x]+[z x y]=0$

rel.

Free alg.

rep. $\mathcal{P}(n)$

$\operatorname{dim} \mathcal{P}(n)$

Gen. series $f(t)$

Dual operad $L T^{!}$

Chain-cplx

Properties ternary, quadratic, Koszul ?.

Alternative the relation admits many different versions due to the symmetry

Relationship $\quad$ Lie-alg $\rightarrow$ LTS-alg, $[x y z]=[[x y] z]$

Comment Appreciate the Leibniz presentation Integration: symmetric spaces

Ref.

[Loo69] Loos O., Symmetric spaces. I. General theory.

W. A. Benjamin, Inc., New York-Amsterdam (1969) viii+198 pp. 
Name

Notation

Def. oper. $\quad x \cdot y, \quad[x, y, z]$

sym. $\quad x \cdot y=-y \cdot x, \quad[x, y, z]=-[y, x, z]$

$[x, y, z]+[y, z, x]+[z, x, y]+(x \cdot y) \cdot z+(y \cdot z) \cdot x+(z \cdot x) \cdot y=0$

rel.

$[x, y, u \cdot v]=u \cdot[x, y, v]+[x, y, u] \cdot v$

$[x, y,[z, t, u]]=[[x, y, z], t, u]-[z,[t, x, y], u]+[z, t,[x, y, u]]$

Free alg.

rep. $\mathcal{P}(n)$

$\operatorname{dim} \mathcal{P}(n)$

Gen. series $f(t)$

Dual operad $L T^{!}$

Chain-cplx

Properties binary and ternary, quadratic, Koszul ?.

Alternative Generalized Lie triple systems

Relationship $\quad L T S$-alg $\rightarrow L Y$-alg, $x \cdot y=0,[x, y, z]=[x y z]$

Unit

Comment

Ref .

[KW01] R. Kinyon, M. Weinstein, A., Leibniz algebras, Courant algebroids, and multiplications on reductive homogeneous spaces. Amer. J. Math. 123 (2001), no. 3, 525-550. 
Name

Notation

Def . oper.

sym.

rel.

\section{Interchange algebra}

Interchange

$x \cdot y, \quad x * y$

$(x \cdot y) *(z \cdot t)=(x * z) \cdot(y * t)$

Free alg.

rep. $\mathcal{P}(n)$

$\operatorname{dim} \mathcal{P}(n)$

Gen. series $f(t)=$

Dual operad

Chain-cplx

Properties binary, cubic, set-theoretic.

Alternative

Relationship Strongly related with the notions of 2-category and 2-group

Unit if a unit for both, then $*=\cdot$ and they are commutative

Comment Many variations depending on the hypotheses on $*$ and . Most common $\cdot$ and $*$ are associative.

Ref . 
Name

Def. oper. sym. rel.

\section{Free alg.}

rep. $\mathcal{P}(n)$

$\operatorname{dim} \mathcal{P}(n)$

Gen. series

Dual operad

Chain-cplx

Properties

Alternative

Relationship

Unit

Comment

Ref.

\section{Hypercommutative algebra}

underlying objects: graded vector spaces

HyperCom

$\left(x_{1}, \ldots, x_{n}\right) n$-ary operation of degree $2(n-2)$ for $n \geq 2$ totally symmetric

$\sum_{S_{1} \sqcup S_{2}=\{1, \ldots, n\}}\left(\left(a, b, x_{S_{1}}\right), c, x_{S_{2}}\right)=$ $\sum_{S_{1} \sqcup S_{2}=\{1, \ldots, n\}}(-1)^{|c|\left|x_{S_{1}}\right|}\left(a,\left(b, x_{S_{1}}, c\right), x_{S_{2}}\right)$, for any $n \geq 0$.

\section{$f(t)=$}

Gravity algebra, see ref below 
Name

Notation

Def. oper. sym. rel.

Free alg.

$\mathcal{P}_{n}$

$\operatorname{dim} \mathcal{P}_{n}$

Gen. series

Dual operad

Chain-cplx

Properties

Alternative

Relationship

Unit

Comment

Ref .

\section{Associative algebra up to homotopy}

operad with underlying space in dgVect

$A_{\infty}$

$m_{n}$ for $n \geq 2$ (operation of arity $n$ and degree $n-2$ )

$\partial\left(m_{n}\right)=\sum_{\substack{n=p+q+r \\ k=p+1+r \\ k>1, q>1}}(-1)^{p+q r} m_{k} \circ\left(\mathrm{id}^{\otimes p} \otimes m_{q} \otimes \mathrm{id}^{\otimes r}\right)$.

$\left(A_{\infty}\right)_{n}=\mathbb{K}\left[P T_{n}\right]$ isomorphic to $C_{\bullet}\left(\mathcal{K}^{n-2}\right)$ as chain complex where $\mathcal{K}^{n}$ is the Stasheff polytope of dimension $n$

$f(t)$

multi-ary, quadratic, ns, minimal model for $A s$

Cobar construction on $A s^{i}: A_{\infty}=A s_{\infty}:=\Omega A s^{i}$

Many, see the literature.

Good question!

There are two levels of morphisms between $A_{\infty}$-algebras: the morphisms and the $\infty$-morphisms, see [LV11] for instance.

[Sta63] J. Stasheff, Homotopy associativity of H-spaces. I, II. TAMS 108 (1963), 275-292 ; ibid. 108 (1963), 293312. 
Name

Def. oper.

sym.

rel.

\section{Commutative algebra up to homotopy}

operad with underlying space in dgVect

$C_{\infty}$

$m_{n}$ for $n \geq 2$ (operation of arity $n$ and degree $n-2$ )

which vanishes on the sum of $(p, n-p)$-shuffles, $1 \leq p \leq n-1$.

Free alg.

\section{$\mathcal{P}(n)$}

$\operatorname{dim} \mathcal{P}(n)$

Gen. series $f(t)$

Dual operad

Chain-cplx

Properties multi-ary, quadratic, minimal model for Com

Alternative Cobar construction on $\mathrm{Com}^{\mathrm{i}}: \mathrm{Com}_{\infty}=\mathrm{Com}_{\infty}:=\Omega \mathrm{Com}^{\mathrm{i}}$

Relationship

Unit

Comment

Ref. $\quad[\operatorname{Kad} 85]$ T. Kadeishvili, The category of differential coalgebras and the category of $A(\infty)$-algebras.

Proc. Tbilisi Math.Inst. 77 (1985), 50-70. 
Name

Notation

Def. oper. sym. rel.

\section{Lie algebra up to homotopy}

operad with underlying space in dgVect

$L_{\infty}$

$\ell_{n}, n$-ary operation of degree $n-2$, for all $n \geq 2$

$\sum_{\substack{p+q=n+1 \\ p, q>1}} \sum_{\sigma \in S h_{p, q}^{-1}} \operatorname{sgn}(\sigma)(-1)^{(p-1) q}\left(\ell_{p} \circ_{1} \ell_{q}\right)^{\sigma}=\partial_{A}\left(\ell_{n}\right)$

Free alg.

rep. $\mathcal{P}(n)$

$\operatorname{dim} \mathcal{P}(n)$

Gen. series $f(t)$

Dual operad

Chain-cplx

Properties multi-ary, quadratic, minimal model for Lie

Alternative Cobar construction on $\operatorname{Lie}^{i}: \operatorname{Lie}_{\infty}=\operatorname{Lie}_{\infty}:=\Omega L i e^{\mathrm{i}}$

Relationship

Unit

Comment

Ref. 
Name

Notation

Def. oper.

sym.

rel.

\section{Dendriform algebra up to homotopy}

operad with underlying space in dgVect

$\operatorname{Dend}_{\infty}$

$m_{n, i}$ is an $n$-ary operation, $1 \leq i \leq n$, for all $n \geq 2$

none

$\partial\left(m_{n, i}\right)=\sum(-1)^{p+q r} m_{p+1+r, \ell}(\underbrace{\mathrm{id}, \cdots, \mathrm{id}}_{p}, m_{q, j}, \underbrace{\mathrm{id}, \cdots, \mathrm{id}}_{r})$

sum extended to all the quintuples $p, q, r, \ell, j$ satisfying:

$p \geq 0, q \geq 2, r \geq 0, p+q+r=n, 1 \leq \ell \leq p+1+q, 1 \leq j \leq q$ and either one of the following:

$i=q+\ell$, when $1 \leq p+1 \leq \ell-1$,

$i=\ell-1+j$, when $p+1=\ell$,

$i=\ell$, when $\ell+1 \leq p+1$.

Free alg.

rep. $\mathcal{P}_{n}$

$\operatorname{dim} \mathcal{P}_{n}$

Gen. series $f(t)=$

Dual operad

Chain-cplx

Properties

multi-ary, quadratic, ns, minimal model for Dend

Alternative

Relationship

Unit

Comment

Ref.

See for instance [LV11] 
Name

Notation

Def. oper.

sym.

rel.

Free alg.

rep. $\mathcal{P}(n)$

$\operatorname{dim} \mathcal{P}(n)$

Gen. series

Dual operad

Chain-cplx

Properties

Alternative

Relationship

Unit

Comment

Ref .

\section{$\mathcal{P}$-algebra up to homotopy}

operad with underlying space in dgVect

$\mathcal{P}_{\infty}$

the operad $\mathcal{P}$ is supposed to be quadratic and Koszul 
Name

Notation

Def . oper. sym. rel.

\section{Brace algebra}

\section{Brace}

$\left\{x_{0} ; x_{1}, \ldots, x_{n}\right\}$ for $n \geq 0$

$\{x ; \emptyset\}=x$

$\left\{\left\{x ; y_{1}, \ldots, y_{n}\right\} ; z_{1}, \ldots, z_{m}\right\}=$ $\sum\left\{x ; \ldots,\left\{y_{1} ; \ldots\right\}, \ldots \ldots,\left\{y_{n} ; \ldots,\right\}, \ldots\right\}$.

the dots are filled with the variables $z_{i}$ 's (in order).

Free alg.

rep. $\mathcal{P}(n)$

$\operatorname{Brace}(n)=\mathbb{K}\left[P B T_{n+1}\right] \otimes \mathbb{K}\left[\mathbb{S}_{n}\right]$

$\operatorname{dim} \mathcal{P}(n)$

$1,1 \times 2 !, 2 \times 3 !, 5 \times 4 !, 14 \times 5 !, 42 \times 6 !, 132 \times 7 !, \ldots, c_{n-1} \times n !, \ldots$

Gen. series

$f(t)$

Dual operad

Chain-cplx

Properties multi-ary, quadratic, quasi-regular.

Alternative

Relationship Brace-alg $\rightarrow M B$-alg

Brace-alg $\rightarrow$ PreLie-alg, $(\{-;-\}$ is a pre-Lie product $)$

If $A$ is a brace algebra, then $T^{c}(A)$ is a cofree Hopf algebra

Unit

Comment

There exists a notion of brace algebra with differentials useful in algebraic topology

Ref.

[Ron02] Ronco, M. Eulerian idempotents and Milnor-Moore theorem for certain non-cocommutative Hopf algebras.

J. Algebra 254 (2002), no. 1, 152-172. 
Name

Notation

Def . oper. sym.

rel. $\mathcal{R}_{i j k}$, see ref

Free alg.

rep. $\mathcal{P}(n)$

$M B(n)=\left(\mathbb{K}\left[P T_{n}\right] \oplus \mathbb{K}\left[P T_{n}\right]\right) \otimes \mathbb{K}\left[\mathbb{S}_{n}\right], n \geq 2$

$\operatorname{dim} \mathcal{P}(n)$

Gen. series

$f(t)$

Dual operad

Chain-cplx

Properties

Alternative

Unit

Comment

Ref .

\section{Multi-brace algebra}

$M B$

$\left(x_{1}, \ldots, x_{p} ; y_{1}, \ldots, y_{q}\right\}$ for $p \geq 1, q \geq 1$ $C_{n}=$ Schröder number (super Catalan)

Relationship Brace-alg $\rightarrow M B$-alg

Brace-alg $\rightarrow$ PreLie-alg, $(\{-;-\}$ is a pre-Lie product $)$ (and vice-versa)

Used to be denoted by $B_{\infty}$ or $\mathbf{B}_{\infty}$ useful in algebraic topology (and called $B_{\infty}$-algebra) If $A$ is a brace algebra, then $T^{c}(A)$ is a cofree Hopf algebra confusing notation with respect to algebras up to homotopy

There exists a notion of MB-infinity algebra with differentials

[LR06] Loday, J.-L., and Ronco, M. On the structure of cofree Hopf algebras J. reine angew. Math. 592 (2006) 123-155. 


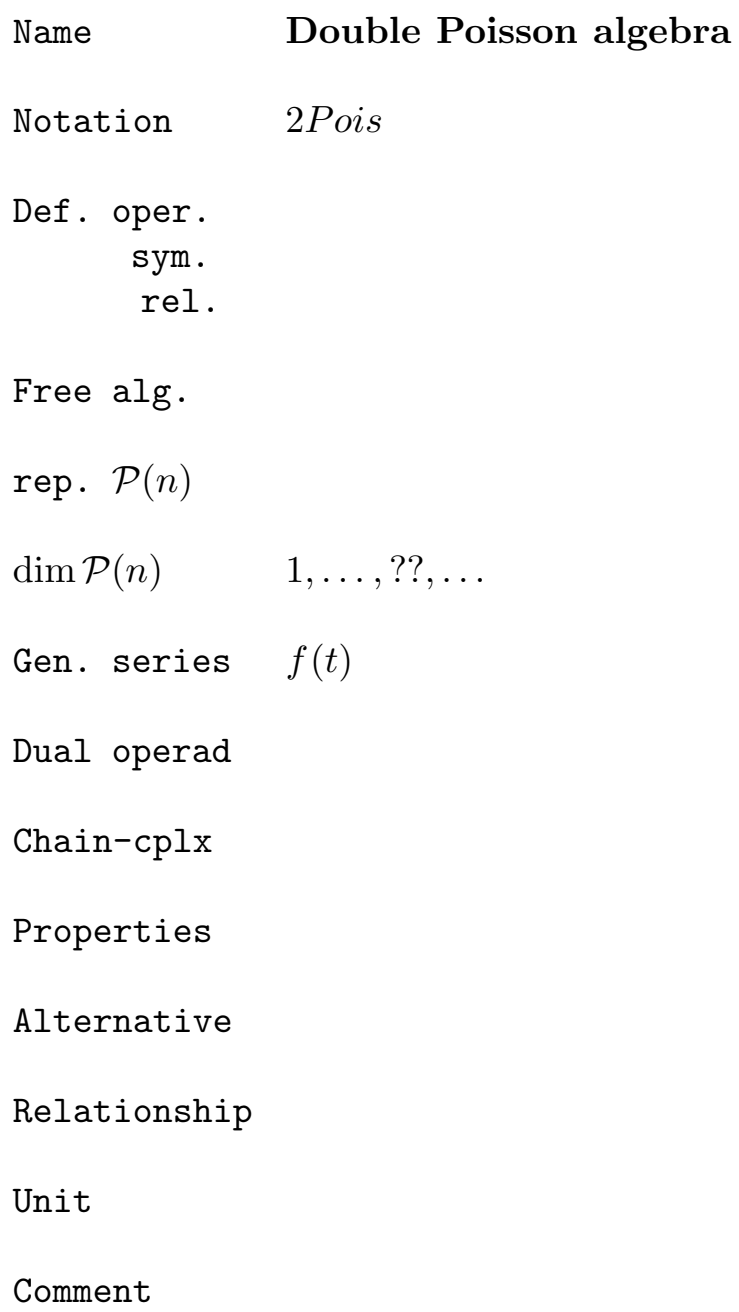

Ref. M. Van den Bergh, 


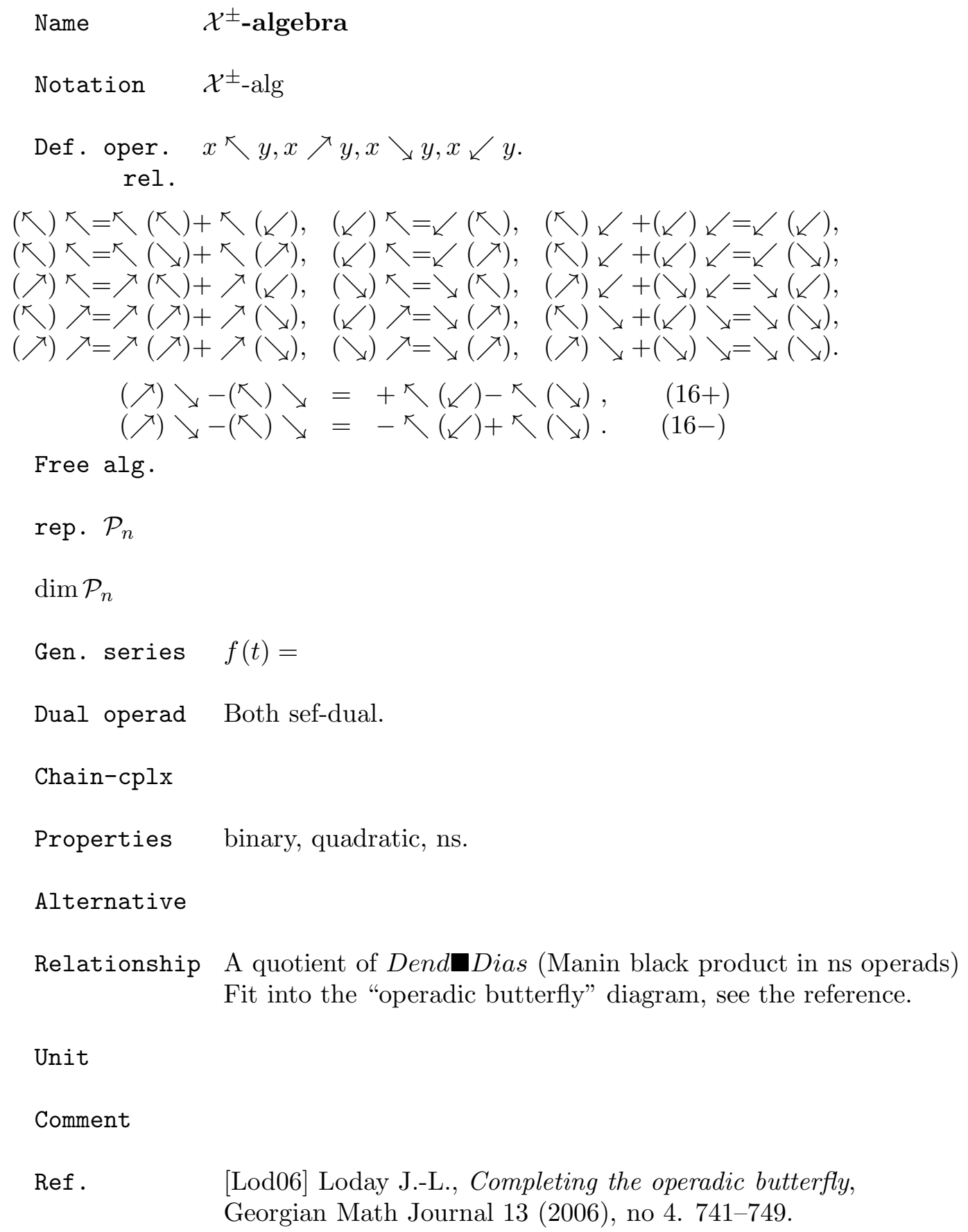


Notation

Def. oper.

sym.

rel.

Free alg.

rep. $\mathcal{P}(n)$

$\operatorname{dim} \mathcal{P}(n)$

Gen. series $f(t)=$

Dual operad

Chain-cplx

Properties

Alternative

Relationship

Unit

Comment

Ref . 
Integer sequences which appear in this paper, up to some shift and up to multiplication by $n$ ! or $(n-1)$ !.

\begin{tabular}{|c|c|c|c|c|c|c|c|c|}
\hline 1 & 1 & 1 & 1 & 1 & $\cdots$ & 1 & $\cdots$ & Com, As \\
\hline 1 & 2 & 0 & 0 & 0 & $\cdots$ & 0 & $\ldots$ & Nil \\
\hline 1 & 2 & 2 & 2 & 2 & $\cdots$ & 2 & $\cdots$ & Dual2as \\
\hline 1 & 2 & 3 & 4 & 5 & $\cdots$ & $n$ & $\cdots$ & Dias, Perm \\
\hline 1 & 2 & 5 & 12 & 15 & $\cdots$ & $? ?$ & $\cdots$ & Altern! \\
\hline 1 & 2 & 5 & 14 & 42 & $\ldots$ & $c_{n-1}$ & 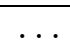 & Mag, Dend,brace, Dup \\
\hline 1 & 2 & 6 & 18 & 57 & $\ldots$ & $f_{n+2}$ & $\ldots$ & MagFine \\
\hline 1 & 2 & 6 & 22 & 90 & $\ldots$ & $2 C_{n}$ & $\ldots$ & Dipt, 2as, brace \\
\hline 1 & 2 & 6 & 24 & 120 & $\cdots$ & $n !$ & 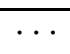 & As, Lie, Leib, Zinb \\
\hline 1 & 2 & 7 & & & $\cdots$ & $? ?$ & $\ldots$ & Lie-adm \\
\hline 1 & 2 & 7 & 32 & 175 & $\cdots$ & $? ?$ & $\ldots$ & Altern \\
\hline 1 & 2 & 7 & 40 & & $\cdots$ & $? ?$ & 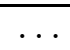 & Moufang \\
\hline 1 & 2 & 9 & 64 & 625 & $\ldots$ & $n^{n-1}$ & $\ldots$ & PreLie, $N A P$ \\
\hline 1 & 2 & 10 & 26 & 76 & $\ldots$ & $? ?$ & $\ldots$ & Parastat \\
\hline 1 & 3 & 7 & 15 & 31 & $\ldots$ & $\left(2^{n}-1\right)$ & $\ldots$ & Trias \\
\hline 1 & 3 & 9 & & & $\cdots$ & $? ?$ & $\cdots$ & Malcev \\
\hline 1 & 3 & 11 & 45 & 197 & $\ldots$ & $C_{n}$ & $\ldots$ & TriDend \\
\hline 1 & 3 & 13 & 75 & 541 & $\cdots$ & $? ?$ & $\ldots$ & $C T D$ \\
\hline 1 & 3 & 16 & 125 & $6^{5}$ & $\cdots$ & $(n+1)^{n-1}$ & $\cdots$ & Park \\
\hline 1 & 3 & 20 & 210 & 3024 & $\cdots$ & $a(n)$ & $\cdots$ & PostLie \\
\hline 1 & 3 & 50 & & & $\cdots$ & $? ?$ & $\cdots$ & Jordan triples \\
\hline 1 & 4 & 9 & 16 & 25 & $\ldots$ & $\overline{n^{2} n !}$ & $\ldots$ & Quadri! \\
\hline 1 & 4 & 23 & 156 & 1162 & $\cdots$ & $? ?$ & $\ldots$ & Quadri \\
\hline 1 & 4 & 23 & 181 & & $\cdots$ & $? ?$ & $\ldots$ & see PreLie \\
\hline 1 & 4 & 27 & 256 & & $\cdots$ & $\overline{n^{n}}$ & $\cdots$ & \\
\hline 1 & 8 & & & & $\cdots$ & $? ?$ & $\ldots$ & Akivis \\
\hline 1 & 8 & 78 & 1104 & & $\cdots$ & $? ?$ & $\ldots$ & Sabinin \\
\hline
\end{tabular}




\section{REFERENCES}

[AL04] Marcelo Aguiar and Jean-Louis Loday, Quadri-algebras, J. Pure Appl. Algebra 191 (2004), no. 3, 205-221.

[Bai10] Guo L. Ni X. Bai, C.M., L-quadri-algebras.

[BF03] Christian Brouder and Alessandra Frabetti, QED Hopf algebras on planar binary trees, J. Algebra 267 (2003), no. 1, 298-322. MR 1993478 (2004m:81160)

[BHP05] Murray R. Bremner, Irvin R. Hentzel, and Luiz A. Peresi, Dimension formulas for the free nonassociative algebra, Comm. Algebra 33 (2005), no. 11, 40634081. MR 2183981 (2006h:17008)

[BL11] N. Bergeron and J.-L. Loday, The symmetric operation in a free pre-Lie algebra is magmatic, Proc. Amer. Math. Soc. (2011).

[Cha01] F. Chapoton, Un endofoncteur de la catégorie des opérades, Dialgebras and related operads, Lecture Notes in Math., vol. 1763, Springer, Berlin, 2001, pp. 105-110.

[Cha02] Frédéric Chapoton, Opérades différentielles graduées sur les simplexes et les permutoèdres, Bull. Soc. Math. France 130 (2002), no. 2, 233-251.

[CL01] F. Chapoton and M. Livernet, Pre-Lie algebras and the rooted trees operad, Internat. Math. Res. Notices (2001), no. 8, 395-408.

[Cov10] S. Covez, The local integration of Leibniz algebras, ArXiv e-prints (2010).

[DK07] V. V. Dotsenko and A. S. Khoroshkin, Character formulas for the operad of a pair of compatible brackets and for the bi-Hamiltonian operad, Funktsional. Anal. i Prilozhen. 41 (2007), no. 1, 1-22, 96.

[Dot09] Vladimir Dotsenko, Compatible associative products and trees, Algebra Number Theory 3 (2009), no. 5, 567-586. MR 2578889 (2010k:18012)

[DZ09] A. Dzhumadil'daev and P. Zusmanovich, The alternative operad is not koszul, arXiv:0906.1272 (2009).

[Fre06] B. Fresse, Théorie des opérades de Koszul et homologie des algèbres de Poisson, Ann. Math. Blaise Pascal 13 (2006), no. 2, 237-312.

[GCTV09] I. Galvez-Carrillo, A. Tonks, and B. Vallette, Homotopy Batalin-Vilkovisky algebras, preprint arXiv:0907.2246 (2009), 49 pp.

[Ger63] M. Gerstenhaber, The cohomology structure of an associative ring, Ann. of Math. (2) 78 (1963), 267-288.

[Get95] E. Getzler, Operads and moduli spaces of genus 0 Riemann surfaces, The moduli space of curves (Texel Island, 1994), Progr. Math., vol. 129, Birkhäuser Boston, Boston, MA, 1995, pp. 199-230.

[GK94] V. Ginzburg and M. Kapranov, Koszul duality for operads, Duke Math. J. 76 (1994), no. 1, 203-272.

[Gne97] Allahtan Victor Gnedbaye, Opérades des algèbres $(k+1)$-aires, Operads: Proceedings of Renaissance Conferences (Hartford, CT/Luminy, 1995), Contemp. Math., vol. 202, Amer. Math. Soc., Providence, RI, 1997, pp. 83-113. MR 1436918 (98a:17003)

[Gon05] A. B. Goncharov, Galois symmetries of fundamental groupoids and noncommutative geometry, Duke Math. J. 128 (2005), no. 2, 209-284. MR 2140264 (2007b:11094)

[HLR08] Ralf Holtkamp, Jean-Louis Loday, and María Ronco, Coassociative magmatic bialgebras and the Fine numbers, J. Algebraic Combin. 28 (2008), no. 1, 97114. MR 2420781 (2009f:16068)

[Kad85] T. V. Kadeishvili, The category of differential coalgebras and the category of $A(\infty)$-algebras, Trudy Tbiliss. Mat. Inst. Razmadze Akad. Nauk Gruzin. SSR 77 (1985), 50-70. MR 862919 (88f:57078) 
[KW01] Michael K. Kinyon and Alan Weinstein, Leibniz algebras, Courant algebroids, and multiplications on reductive homogeneous spaces, Amer. J. Math. 123 (2001), no. 3, 525-550. MR 1833152 (2002d:17004)

[Ler04] Philippe Leroux, Ennea-algebras, J. Algebra 281 (2004), no. 1, 287-302.

[Ler11] _ Hochschild two-cocycles and a good triple of operads, Int. Electron. J. Algebra (2011).

[Liv06] Muriel Livernet, A rigidity theorem for pre-Lie algebras, J. Pure Appl. Algebra 207 (2006), no. 1, 1-18.

[Lod93] Jean-Louis Loday, Une version non commutative des algèbres de Lie: les algèbres de Leibniz, Enseign. Math. (2) 39 (1993), no. 3-4, 269-293.

[Lod95] Cup-product for Leibniz cohomology and dual Leibniz algebras, Math. Scand. 77 (1995), no. 2, 189-196.

[Lod01] Dialgebras, Dialgebras and related operads, Lecture Notes in Math., vol. 1763, Springer, Berlin, 2001, pp. 7-66.

[Lod04] Scindement d'associativité et algèbres de Hopf, Actes des Journées Mathématiques à la Mémoire de Jean Leray, Sémin. Congr., vol. 9, Soc. Math. France, Paris, 2004, pp. 155-172.

[Lod05] _ Inversion of integral series enumerating planar trees, Sém. Lothar. Combin. 53 (2005), Art. B53d, 16 pp. (electronic). MR MR2180779 (2006k:05016)

[Lod06] Completing the operadic butterfly, Georgian Math. J. 13 (2006), no. 4, $741-749$.

[Lod07] On the algebra of quasi-shuffles, Manuscripta Math. 123 (2007), no. 1, 79-93.

[Lod08] _ Generalized bialgebras and triples of operads, Astérisque (2008), no. $320, \mathrm{x}+116$.

[Loo69] Ottmar Loos, Symmetric spaces. I: General theory, W. A. Benjamin, Inc., New York-Amsterdam, 1969. MR 0239005 (39 \#365a)

[LR03] Jean-Louis Loday and María Ronco, Algèbres de Hopf colibres, C. R. Math. Acad. Sci. Paris 337 (2003), no. 3, 153-158. MR 2001126 (2004g:16041)

[LR04] Trialgebras and families of polytopes, Homotopy theory: relations with algebraic geometry, group cohomology, and algebraic $K$-theory, Contemp. Math., vol. 346, Amer. Math. Soc., Providence, RI, 2004, pp. 369-398. MR 2066507 (2006e:18016)

[LR06] On the structure of cofree Hopf algebras, J. Reine Angew. Math. 592 (2006), 123-155.

[LV11] Jean-Louis Loday and Bruno Vallette, Algebraic operads, 2011.

[OS06] A. V. Odesskii and V. V. Sokolov, Integrable matrix equations related to pairs of compatible associative algebras, J. Phys. A 39 (2006), no. 40, 12447-12456. MR 2265833 (2007f:17042)

[PI07] José M. Pérez-Izquierdo, Algebras, hyperalgebras, nonassociative bialgebras and loops, Adv. Math. 208 (2007), no. 2, 834-876. MR 2304338 (2008f:17006)

[PIS04] José M. Pérez-Izquierdo and Ivan P. Shestakov, An envelope for Malcev algebras, J. Algebra 272 (2004), no. 1, 379-393. MR 2029038 (2004j:17040)

[Ron00] María Ronco, Primitive elements in a free dendriform algebra, New trends in Hopf algebra theory (La Falda, 1999), Contemp. Math., vol. 267, Amer. Math. Soc., Providence, RI, 2000, pp. 245-263.

[Ron02] _ Eulerian idempotents and Milnor-Moore theorem for certain noncocommutative Hopf algebras, J. Algebra 254 (2002), no. 1, 152-172.

[She04] Ivan P. Shestakov, Moufang loops and alternative algebras, Proc. Amer. Math. Soc. 132 (2004), no. 2, 313-316 (electronic). MR 2022350 (2004j:20126)

[Sta63] James Dillon Stasheff, Homotopy associativity of H-spaces. I, II, Trans. Amer. Math. Soc. 108 (1963), 275-292; ibid. 108 (1963), 293-312. 
[Val07] B. Vallette, A Koszul duality for props, Trans. of Amer. Math. Soc. 359 (2007), 4865-4993.

[Val08] Bruno Vallette, Manin products, Koszul duality, Loday algebras and Deligne conjecture, J. Reine Angew. Math. 620 (2008), 105-164.

[Vin63] È. B. Vinberg, The theory of homogeneous convex cones, Trudy Moskov. Mat. Obšč. 12 (1963), 303-358.

Zinbiel Institute of Mathematics (France)

E-mail address: gw.zinbiel@free.fr 